Nonlinear Fourier Analysis for Unmagnetized Plasma Waves

This content has been downloaded from IOPscience. Please scroll down to see the full text. 2003 Phys. Scr. 687

(http://iopscience.iop.org/1402-4896/68/1/001)

View the table of contents for this issue, or go to the journal homepage for more

Download details:

IP Address: 146.175.11.111

This content was downloaded on 09/12/2013 at 13:53

Please note that terms and conditions apply. 


\title{
Nonlinear Fourier Analysis for Unmagnetized Plasma Waves
}

\author{
D. K. Callebaut* and G. K. Karugila**广 \\ Physics Department, UIA, University of Antwerp, B-2610 Antwerp, Belgium. \\ Received October 28, 2002; revised version received February 26, 2003; accepted March 4, 2003 \\ PACS Ref: $02.30 . J r, 02.30 . \mathrm{Nw}, 52.35 .-\mathrm{g}$, 52.35.Fp.
}

\begin{abstract}
We apply the nonlinear Fourier analysis developed by Callebaut [1] to an infinite homogeneous plasma calculating many higher order terms (computer algebra) and obtaining in this way some analytic expressions. (a) For cold plasma: the maximum amplitude is $2 / e$ (i.e., $73 \%$ of $n_{0}$ ) of the initial density $n_{0}$, otherwise the series diverges. For exponentials (sum of two waves) the maximum amplitude is halved, i.e $n_{0} / e$. (b) For plasma with electron pressure, the radius of convergence decreases as the ratio of $k^{2} v_{s-}^{2}\left(1+\Gamma_{-}\right) / \omega_{-}^{2}$ increases ( $\Gamma_{-}$is the polytropic exponent; $\omega_{-}$is the plasma angular frequency for electrons; $k$ is the wave number; $v_{s-}$ is the sound velocity for the electrons). (c) Suggestions for experimental verification are made. (d) In the limit of sound waves (no plasma) the radius of convergence is zero. Nevertheless the correct dispersion relation is obtained. A direct analysis confirmed these results for sound waves. (e) The cases where the method fails are indicated. (f) Plasma where both ions and electrons may move, are briefly considered (relevant for comet tails, fullerenes and electron-positron plasmas).
\end{abstract}

\section{Introduction}

In the previous decades a lot of work on nonlinear theory of plasma waves and instabilities has been done. We may mention the papers with the exact solutions by Malfliet et al. [2], Hereman et al. [3], Verheest et al. [4] on solitary waves and those of Khater et al. [5] on Bäcklund transformations, on the AKNS system and the Painlevé analysis. Recently Amiranashvili et al. [6] gave some exact solutions for standing waves in bounded plasmas without using the solitary wave theory but with some boundary conditions. Such solutions may be quite interesting in themselves and very useful to verify numerical calculations. Callebaut and Tsintsadze, e.g., neglecting some higher order terms, dealt with the nonlinear bunching of Alfvén waves [7]. In fact, except for the approaches leading to solitary wave solutions, the nonlinear methods usually yield approximations and usually one has barely an idea how long these are valid in the behavior of the plasma. The present approach exploits the Fourier analysis for nonlinear systems. It is rather different from the approaches just mentioned as it allows some insight in the convergence. Moreover, it gives useful results for the many cases where one can not find a closed form for the solitary waves.

The set of (partial differential) equations (e.g., equations (1), (2) and (3)), together with some initial and/or boundary conditions, defines a set of functions (which are, of course, interrelated). From Fourier theory it is known that if a periodic function is continuous from $-\infty$ to $+\infty$ and has a derivative which is piecewise monotonous

\footnotetext{
* e-mail: dirk.callebaut@ua.ac.be

** e-mail: geoffrey.karugila@ua.ac.be

${ }^{\dagger}$ On leave from Department of Mathematics and Biometry, Faculty of Science, SUA, Morogoro, Tanzania.
}

and continuous, then the function may be developed in a Fourier series which is absolutely and uniformly convergent in any interval. (Some conditions like those of Dirichlet, are even more general). In our work we deal with the single variable $\chi(=\omega t+\boldsymbol{k} \cdot \boldsymbol{r})$, which is the combination of four independent variables, i.e., the angular frequency $(\omega=2 \pi v, v=$ frequency), the time $(t)$, the wave vector $(\boldsymbol{k})$ and space $(\boldsymbol{r})$. Thus the conditions have to be satisfied for the function(s) of this combined variable. In particular the function should be periodic in $\omega t$ and in $\boldsymbol{k} \cdot \boldsymbol{r}$. However, an exponential growth is easily accommodated like the periodic situations as was the case in [1]. Hence under rather general conditions it is possible to expand the functions defined by the set of equations. When will this break down? e.g., when the series diverges, i.e., physically speaking, when instability develops or when an infinite amount of energy has been made available (either injected externally or freed by the system itself from, e.g., its potential energy). The convergence of the series puts conditions on the linear theory, mainly on its amplitude. In fact a linearized theory can never determine its own limitations: that has to be done by the nonlinear analysis. In the previous works [1] it turned out that some experimental situations, in particular the oscillation and instabilities of a liquid jet, could be explained very well by this method. Moreover, some cases appeared where the nonlinear theory showed that the linear theory was good even up to breakdown of the configuration [1]. It may be noted that [1] was more general as the four variables were treated independently and not combined into the single variable $\chi$; moreover [1] dealt with instability too. However, for the problem at hand (no boundaries, plasma waves) this is rather irrelevant.

Another breakdown of the method may occur, e.g., when the function(s) is (are) not periodic. (Nevertheless instabilities may be included, but they are not relevant in the present paper). However, in the linear perturbation theory one works usually with a periodic perturbation and this generates naturally higher order terms which are periodic too as is obvious, e.g., in our present work and in the work of Callebaut, e.g. [1,8]. For a non-periodic solution one has to take a wholly different start in the linear theory, e.g., by using a series in $t$ and/or $x, y, z$, or some adequate combination of those, or if nevertheless a periodic or exponential start is used as first order term, to adapt profoundly the nonlinear terms. Such nonlinear approaches have been elaborated in various ways in the literature, see e.g. [2-5, 7]. Those methods are indicated in the cases that the nonlinear Fourier analysis is not adequate, e.g., when considering sound waves (Section 3.2). 
The plan of the paper is as follows: In Section 2, we give the basis, from which we formulate a model, state the basic equations and consider the equilibrium case. In Section 3 we study the unmagnetized plasma for which the ion particle density is not perturbed. We therefore break this section into three subsections, namely cold plasma, sound waves and plasma with pressure of electrons. In each subsection, we first reduce the system of partial differential equations to one equation, using at least two different procedures. This is followed by a perturbation analysis, from which we calculate several coefficients to higher orders and in some cases, obtain analytic expressions. In Section 4 the case of unmagnetized plasma with perturbed ion particle density is investigated. Here we reduce the system of equations to two partial differential equations from which we determine the coefficients up to order five. Some attention is given to electron-positron plasma. Finally the paper ends with a conclusion in Section 5.

We have elaborated various alternative procedures for simple cases like cold plasma and sound waves. The reasons for this are:

(a) some procedures seemed to lead to contradictions unless extreme care was applied,

(b) to find out which procedure was the simplest and the safest, and

(c) sound waves are baffling because of zero convergence.

\section{Basis}

\subsection{Model}

We consider a fully ionized plasma in a medium infinite in all directions (no boundary conditions) and at rest, i.e., we consider an equilibrium configuration in which perturbations (not necessarily small ones) are generated. The particles are ionized only once and no source terms are considered (no particle creation or recombination). Viscosity, resistivity, gravitation and the magnetic contribution are neglected.

\subsection{Basic equations}

The basic equations are the continuity equations for the charge and for the matter, the equations of motion, the Poisson equation and the polytropic equation. Thus, if there is only one kind of positive ions present, the basic system of equations is written [9]

$\partial_{t} n_{ \pm}+\operatorname{div}\left(n_{ \pm} \boldsymbol{v}_{ \pm}\right)=0$

$n_{ \pm} m_{ \pm} \frac{\mathrm{d} \boldsymbol{v}_{ \pm}}{\mathrm{d} t}=-\nabla p \mp e n_{ \pm} \nabla \varphi$,

$\Delta \varphi=e\left(n_{-}-n_{+}\right) / \varepsilon$,

$p=p_{+}+p_{-}$,

$p_{ \pm}=K_{ \pm}\left(n_{ \pm}\right)^{\Gamma_{ \pm}}$,

where $n_{+}$and $n_{-}$are the respective densities of the positive and negative particles, $\boldsymbol{v}_{-}$and $\boldsymbol{v}_{+}$are the fluid velocities of electrons and ions respectively, $e=1.6 \times 10^{-19} \mathrm{C}$ and $-e$ are the respective charges of the positive ion (with mass $m_{+}=1.67 \times 10^{-27} \mathrm{~kg}$ for proton) and the negative ion, usually the electron (with mass $m_{-}=0.91 \times 10^{-30} \mathrm{~kg}$ ), $\varphi$ the electrical potential, $p$ the total pressure, $p_{+}$and $p_{-}$are partial pressures of the ions and electrons respectively, $\varepsilon$ is the permittivity, which for vacuum is $8.85 \times 10^{-12} \mathrm{C} / \mathrm{Vm}$ and $K_{+}, K_{-}, \Gamma_{+}$and $\Gamma_{-}$are constants. The indices + and - are used systematically for positive particles (usually ions; in some cases positrons) and negative particles (usually electrons).

\subsection{Equilibrium}

At equilibrium, plasma is at rest. Thus using a subscript zero for indicating quantities at equilibrium, we put $\boldsymbol{v}_{+0}=\boldsymbol{v}_{-0}=\boldsymbol{v}_{0}=\mathbf{0}$. Plugging these into our basic system of equations, we see that $n_{ \pm 0}, p_{0}$ and $p_{ \pm 0}$ are constants which are independent of time and space. Quasi-neutrality $\left(n_{+0}=n_{-0}=n_{0}\right)$ follows from equilibrium.

\section{Plasma with unperturbed ion density}

Usually ions are much heavier than electrons. The customary assumption is that ions are not moving (in comparison to the electrons). The generalization to perturbed ion density, which is necessary for certain plasmas, will be considered in Section 4. Hence introducing the approximation $n_{+}=n_{0}$, meaning that the ion density is unperturbed, one may consider the following cases:

\subsection{Cold plasma}

Here we suppose that the kinetic motion of the charged particles is negligible and thus the temperature is low enough to be approximated by zero in consideration with other effects (in the present case electric ones). Hence our basic system of equations is reduced to

$\partial_{t} n_{-}+\operatorname{div}\left(n_{-} \boldsymbol{v}_{-}\right)=0$

$n_{-} m_{-} \frac{\mathrm{d} \boldsymbol{v}_{-}}{\mathrm{d} t}=e n_{-} \nabla \varphi$

$\Delta \varphi=e\left(n_{-}-n_{0}\right) / \varepsilon$.

Assume that the phase velocity of the wave (i.e., $\omega / k$ ) is the same for all terms. Physically speaking there is no dispersion between the terms of one family generated by a solution of the linearized equation as far as the phase velocity is concerned (All phases travel with the same speed, nothing is said about the group velocity). This assumption is very plausible as the linearized solution generates in the nonlinear equations higher orders with a multiple of $\chi=\omega t+\boldsymbol{k} \cdot \boldsymbol{r}$. (In fact these are integer multiples, which makes the full solution actually periodic if the first order term is periodic, which is often the case; moreover instabilities may easily be accommodated). Clearly we restrict ourselves to one $\chi$ and its multiples, which form a family. If in the initial perturbation two $\chi$ 's occur, not multiples of each other, then each one will have its own family with its proper $\chi$ and moreover interference terms will occur. We then restrict our analysis here to the case that quantities are functions of $\chi$ alone. This makes it possible to write $\partial_{t} X \equiv \omega \partial_{\chi} X=\omega X^{\prime}$ and $\nabla X \equiv \boldsymbol{k} \partial_{\chi} X=$ $\boldsymbol{k} X^{\prime}$, where the accent means derivative with respect to $\chi$ 
and $X$ stands for $n_{-}, \boldsymbol{v}_{-}$or $\varphi$. Applying these to Eqs, (6)-(8) we have

$\omega n_{-}^{\prime}+\boldsymbol{k} \cdot\left(n_{-} \boldsymbol{v}_{-}\right)^{\prime}=0$,

$m_{-}\left(\omega+\boldsymbol{v}_{-} \cdot \boldsymbol{k}\right) \boldsymbol{v}_{-}^{\prime}=e \boldsymbol{k} \varphi^{\prime}$,

$k^{2} \varphi^{\prime \prime}=e\left(n_{-}-n_{0}\right) / \varepsilon$.

We then eliminate $\boldsymbol{v}_{-}$and $\varphi$ from (9)-(11) in order to reduce the system to a single equation as follows: Integrate (9) to obtain

$\left(\omega+\boldsymbol{k} \cdot \boldsymbol{v}_{-}\right) n_{-}=\epsilon_{-}$.

$\epsilon_{-}$is a constant. Using equilibrium quantities we have

$\epsilon_{-}=\omega n_{0}$.

Substituting this into (9) yields

$\boldsymbol{k} \cdot \boldsymbol{v}_{-}^{\prime}=-\omega n_{0} n_{-}^{\prime} / n_{-}^{2}$

which is then plugged into (10) (taken with dot product with $\boldsymbol{k}$ ) to obtain

$\varphi^{\prime}=-\left(m_{-} \omega^{2} n_{0}^{2} n_{-}^{\prime}\right) /\left(e k^{2} n_{-}^{3}\right)$

We then finish our process of reducing the system to a single equation by using one of the following procedures:

Procedure 1: (Reduction to nonlinear differential equation of first order).

Multiply $\varphi^{\prime}$ on both sides of Eq. (11) and then integrate to have

$\varphi^{\prime 2}+\subset_{1}=\frac{2 e}{\varepsilon k^{2}} \int\left(n_{-}-n_{0}\right) \varphi^{\prime} \mathrm{d} \chi$,

which yields, after substituting (15) into it, the following equation:

$\left(-\frac{m_{-} \omega^{2} n_{0}^{2} n_{-}^{\prime}}{e k^{2} n_{-}^{3}}\right)^{2}+C_{1}=\frac{m_{-} \omega^{2} n_{0}^{2}}{\varepsilon k^{4} n_{-}^{2}}\left(2 n_{-}-n_{0}\right)$.

We then determine $\subset_{1}$ by substituting the lowest order of $n_{-}$into this equation. Hence, Eq. (16) becomes

$n_{-}^{\prime 2}=-\omega_{-}^{2}\left(n_{-}-n_{0}\right)^{2} n_{-}^{4} /\left(\omega^{2} n_{0}^{4}\right)$,

where $\omega_{-}^{2}=e^{2} n_{0} /\left(m_{-} \varepsilon\right)$ is the square of the electron plasma frequency. Taking square roots on both sides we have

$n_{-}^{\prime}= \pm \sqrt{\frac{-\omega_{-}^{2}}{\omega^{2} n_{0}^{4}}}\left(n_{-}-n_{0}\right) n_{-}^{2}$

Due to reasons to be pointed out later, we will reject an equation involving a negative sign in front of the square root. As we will use complex values for $n_{-}$the - sign under the square root is harmless.

Procedure 2: (Reduction to nonlinear differential equation of second order).
Differentiate (15) with respect to $\chi$ to obtain

$\varphi^{\prime \prime}=m_{-} \omega^{2} n_{0}^{2}\left(3 n_{-}^{\prime 2}-n_{-} n_{-}^{\prime \prime}\right) /\left(e k^{2} n_{-}^{4}\right)$.

Substituting (19) into (11) and rearranging we have

$3 n_{-}^{\prime 2}-n_{-} n_{-}^{\prime \prime}+\frac{\omega_{-}^{2} n_{-}^{4}}{\omega^{2} n_{0}^{3}}\left(n_{0}-n_{-}\right)=0$.

Procedure 3: (Reduction to a fully integrated equation).

Equation (18) may be integrated by using the method of separation of variables to give:

$\frac{1}{n_{0}^{2}} \ln \left(1-\frac{n_{0}}{n_{-}}\right)+\frac{1}{n_{-} n_{0}}+\subset_{2}-\frac{i \chi \omega_{-}}{\omega n_{0}^{2}}=0$.

However the determination of the constant $\subset_{2}$ requires care. Putting $n_{-}=n_{0}$ yields $-\infty$ for $\ln \left(1-n_{0} / n_{-}\right)$. Hence to fix $\subset_{2}$ we have to include the first order perturbation, see (26):

Linearizing yields

$\frac{1}{n_{0}^{2}}\left(1-\frac{2 n_{-1}}{n_{0}}+\ln \frac{n_{-1}}{n_{0}}\right)+\subset_{2}-\frac{\mathrm{i} \chi \omega_{-}}{\omega n_{0}^{2}}=0$

Putting (25) (see below) into (22) and simplifying yields for the zero order terms

$\ln A_{-}+1+\subset_{2} n_{0}^{2}+\mathrm{i} \chi\left(1-\left(\omega_{-} / \omega\right)\right)=0$,

which is an expression involving real and imaginary parts. The real part fixes $\subset_{2}$, i.e., $\subset_{2}=-\left(1+\ln A_{-}\right) / n_{0}^{2}$, and the imaginary part gives, if and only if $\chi \neq 0$, the well-known dispersion relation relation for cold plasma:

$\omega=\omega_{-}$.

Equation (21) therefore becomes

$\left(1-\left(n_{0} / n_{-}\right)\right) \mathrm{e}^{n_{0}-n_{-}}=A_{-} \mathrm{e}^{1+\mathrm{i} \chi}$,

which is our fully integrated equation. This shows that $n_{-} / n_{0}$ is a function of exp $\mathrm{i} \chi$, thus a series in exp $\mathrm{i} \chi$ is indicated, see (26) below.

Comment: Ultimately all four nonlinear equations (17), (18), (20) and (24) have to be equivalent (taking into account initial conditions). Equations (17) and (18) are differential equations of first order, Eq. (20) is of second order and Eq. (24) is fully integrated. It is our purpose to show that they lead indeed to the same results, using utmost care, and to find out which procedure is the easiest for future use.

\subsubsection{Analysis of perturbation using exponentials}

(I) Particle density. As we explained above, after equation (8), the solution will usually be periodic if the solution of the linearized equation is periodic, which is the commonly accepted situation for this kind of plasma waves. We also know that it is possible for a periodic motion to be decomposed by Fourier analysis into a superposition of sinusoidal oscillations with different wavelengths and frequencies. If the oscillation amplitude 
is small, then the waveform may be approximated as sinusoidal and one may work with only one component. Hence we let a single Fourier term (cf. the explanation given before Eq. (9)) to be our first order perturbation and we put

$n_{-1}=A_{-} n_{0} \mathrm{e}^{\mathrm{i} \chi}$,

where $A_{-}$is the initial amplitude of the first order perturbation (relative to the equilibrium density). Later on, the upper limit of $A_{-}$or radius of convergence of the series will be determined; this is the maximum value of $A_{-}$ for which our series development can be valid. The general form of the particle density associated with the first order term (25) can, in view of our considerations, be written as

$n=n_{0}+n_{-1}+n_{-2}+\cdots=n_{0} \sum_{s} \alpha_{-s \rho} A_{-}^{s} \mathrm{e}^{\mathrm{i} s \chi}$,

where $a_{-0 \rho}=a_{-1 \rho}=1$. Notice that we have used here $\rho$ as one of the subscripts. In this notation it is not a function. Hence from now on-wards, all $\rho$ 's, $v$ 's, $\varphi$ 's and $f$ 's appearing in the subscripts, should be considered as symbols and not as functions. They are used in the subscripts just to indicate the quantities to which the coefficient belongs. For example, $a_{-1 \rho}$ is the coefficient in the first order term of the negative (normally electrons) particle density expression (cold plasma case) and $c_{-1 \rho}$ is the coefficient in the first order term of the negative particle density expression which will be considered in section 4 . The values of $a_{-s \rho}$, where $s=2,3, \ldots$, are determined by solving either one of Eqs. (17), (18), (20) or (24) as illustrated below:

\section{Determination of $a_{-s \rho}$ 's using Eq. (18)}

1. Substitute $n=n_{0}+n_{-1}=n_{0}\left(1+A_{-} \mathrm{e}^{\mathrm{i} \chi}\right)$ into (18), linearize and rearrange to get (23). Note that we have rejected the solution with - sign,

$\omega=-\omega_{-} n_{0}$

which is false because it contradicts (23). This false result was probably introduced when we multiplied Eq. (11) by $\varphi^{\prime}$ in procedure 1 above. Thus, we reject in Eq. (18) the negative sign in front of the square root. We have a confirmation for this rejection in the next step.

2. We then use (26) (up to order two) in (18) to obtain

$$
1+2 \alpha_{-2 \rho} A_{-} \mathrm{e}^{\mathrm{i} \chi}= \pm\left(1+\left(2+a_{-2 \rho}\right) A_{-} \mathrm{e}^{\mathrm{i} \chi}\right) .
$$

If the right-hand side of (28) has a + sign, then $a_{-2 \rho}=2$.
On the other hand, if the right-hand side has a - sign, $a_{-2 \rho}=-2\left(1+A_{-} \exp (\mathrm{i} \chi)\right) /\left(3 A_{-} \exp (\mathrm{i} \chi)\right)$. We reject this since it depends on $\chi$. We therefore take $a_{-2 \rho}=2$ because it is a constant independent of $\chi$. Following these steps, we wrote a program in mathematica [10] to solve for the remaining values of $a_{-s \rho}$. In Table I we show some of the obtained values of $a_{-s \rho}$. Examining these values very closely, we infer the following analytic expression

$a_{-s \rho}=s^{s} / s !$

Putting (29) into (26) we get

$$
n_{-}=n_{0}\left(1+\sum_{s=1}^{N} \frac{s^{s}}{s !} A_{-}^{s} \mathrm{e}^{\mathrm{i} s \chi}\right)
$$

which converges when $\left|A_{-}\right|<1 / \mathrm{e}$, according to d'Alembert ratio test [11, p. 45].

Determination of $a_{-s \rho}$ 's using Eq. (17), Eq. (20) or Eq. (24):

Here the previous result is confirmed. However equation (20) produced results faster than the other equations, thus we conclude that the procedure using the differential equation of the highest order is quicker and shorter than the other two.

(II) Velocity. The velocity terms are investigated by solving Eq. (12) as elaborated below:

Substituting for $n_{-}$and $\boldsymbol{v}_{-}$in (12) where $\boldsymbol{v}_{-}$, in agreement with the series for $n_{-}$, reads:

$\boldsymbol{v}_{-}=\boldsymbol{v}_{-1}+\boldsymbol{v}_{-2}+\cdots=\frac{\boldsymbol{k}}{k} \sum_{s} a_{-s v} A_{-}^{s} \mathrm{e}^{\mathrm{i} s \chi}$

with constants $a_{-s v}$ to be determined. In Table I, we show the first ten values of $a_{-s v}$. From these values, we inferred the analytic expression

$a_{-s v}=-s^{s-1} v_{\varphi} / s !$

where $v_{\varphi}=\omega / k$ has the dimensions of velocity. We then put this into (31) and tested for convergence using d'Alembert's test. We found out that, the series is convergent when $\left|A_{-}\right|<\mathrm{e}^{-1}$, in harmony with the convergence of the series for the particle density.

Table I. Table showing, for cold plasma case, values of the first ten dimensionless coefficients (i.e., $\left.\mathrm{a}_{-0 \rho}, \mathrm{a}_{-1 \rho}, \ldots, \mathrm{a}_{-10 \rho}\right)$ in the density expression. It also shows the values of the first ten coefficients in the velocity expression (i.e.,

\begin{tabular}{|c|c|c|c|c|c|c|c|c|c|c|c|}
\hline$s$ & 0 & 1 & 2 & 3 & 4 & 5 & 6 & 7 & 8 & 9 & 10 \\
\hline$a_{-s \rho}$ & 1 & 1 & 2 & $\frac{9}{2}$ & $\frac{32}{3}$ & $\frac{625}{24}$ & $\frac{324}{5}$ & $\frac{117649}{720}$ & $\frac{131072}{315}$ & $\frac{4782969}{4480}$ & $\frac{1562500}{567}$ \\
\hline$a_{-s v}$ & 0 & -1 & -1 & $-\frac{3}{2}$ & $-\frac{8}{3}$ & $-\frac{125}{24}$ & $-\frac{54}{5}$ & $-\frac{16807}{720}$ & $-\frac{16384}{315}$ & $-\frac{531441}{4480}$ & $-\frac{156250}{567}$ \\
\hline$a_{-s \varphi}$ & 0 & -1 & $-\frac{1}{2}$ & $-\frac{1}{2}$ & $-\frac{2}{3}$ & $-\frac{25}{24}$ & $-\frac{9}{5}$ & $-\frac{2401}{720}$ & $-\frac{2048}{315}$ & $-\frac{59049}{4480}$ & $-\frac{15625}{567}$ \\
\hline
\end{tabular}
$\mathrm{a}_{-0 v}, \mathrm{a}_{-1 \mathrm{v}}, \ldots, \mathrm{a}_{-10 \mathrm{v}}$, in units $\mathrm{v}_{\varphi}=\omega / \mathrm{k}$ ) and those of the potential expression (i.e., $\mathrm{a}_{-0 \varphi}, \mathrm{a}_{-1 \varphi}, \ldots, \mathrm{a}_{-10 \varphi}$, in units en $\mathrm{n}_{0} / \mathrm{k}^{2} \varepsilon$ ). 
(III) Potential. Here we integrate (15) with respect to $\chi$ to get

$\varphi+\subset_{3}=m_{-} \omega^{2} n_{0}^{2} /\left(2 e k^{2} n_{-}^{2}\right)$.

By substituting the lowest order quantities into this we determine $\subset_{3}$ and hence Eq. (32) becomes

$\varphi=m_{-} \omega^{2}\left(n_{0}^{2}-n_{-}^{2}\right) /\left(2 e k^{2} n_{-}^{2}\right)$

In view of the Fourier analysis for $n_{-}$we take

$\varphi=\sum_{s} a_{-s \varphi} A_{-}^{s} \mathrm{e}^{\mathrm{i} s \chi}$

Then, we substitute this into (33) and solve for $a_{-s \varphi}$ to obtain the results shown in Table I. From these results, we infer the following general expression:

$a_{-s \varphi}=-\frac{s^{s-2}}{s !} \frac{e n_{0}}{k^{2} \varepsilon}$

This has the dimensions of an electric potential.

As in the previous analysis, the series in (34) with (35) converges when $\left|A_{-}\right|<\mathrm{e}^{-1}$ in harmony with the convergence of the series for the particle density and the velocity.

\subsubsection{Graphical results}

\section{Convergence}

The analytical results shows that our series will only converge if $\left|A_{-}\right|<1 /$ e. In order to visualize this we sketch

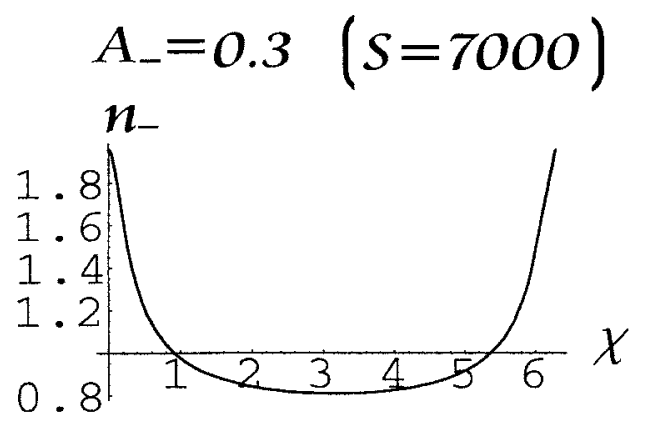

$$
A_{-}=0.37 \quad(S=300)
$$

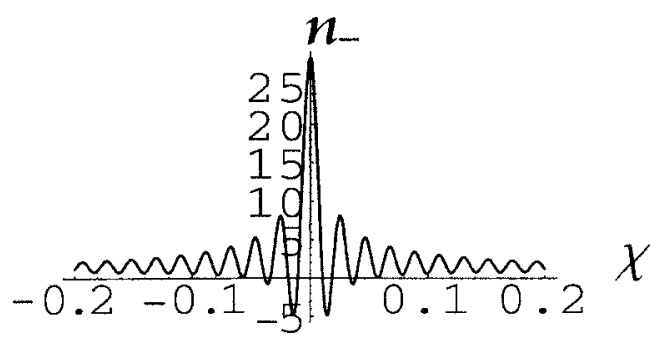

some graphs involving $A_{-}>1 / \mathrm{e}$ (divergence case) and some involving $\left|A_{-}\right|<1 / \mathrm{e} \quad$ (convergence case), see Fig. 1.

From the graphs we note that when $\left|A_{-}\right|<1 / \mathrm{e}$, the minimum points for $n_{-}$in the graphs never go below zero (based on the result of up to order 7000). But for the cases where $A_{-}>1 / \mathrm{e}$, some points are below zero. Negative density is excluded because of the conservation of particles and charge and because of the positive uniform background. We therefore conclude that, if at least one of the minimum points is negative, then the series is invalid or divergent for all values greater or equal to the chosen $A_{-}$. But if all the minimum points for $n_{-}$ are positive then the series is convergent for $A_{-}$equal or less than the selected value. It is nice to see that these numerical observations coincide with the result of the analytic considerations.

We note that for a cold plasma the convergence is independent of $k$, in contrast to the situation with pressure or the cases treated by Callebaut $[1,8]$.

\section{Conservation of particles}

We also calculated the area under the curve of $n_{-}-n_{0}$ against $\chi$ (only real values of $n_{-}$in (26) are being considered) and found out that for the first order case, the area is zero. When higher orders are included in the series, the area obtained ranged between $1 \times 10^{-14}$ and $1 \times 10^{-16}$. This shows that the number of particles is conserved within the computational error.
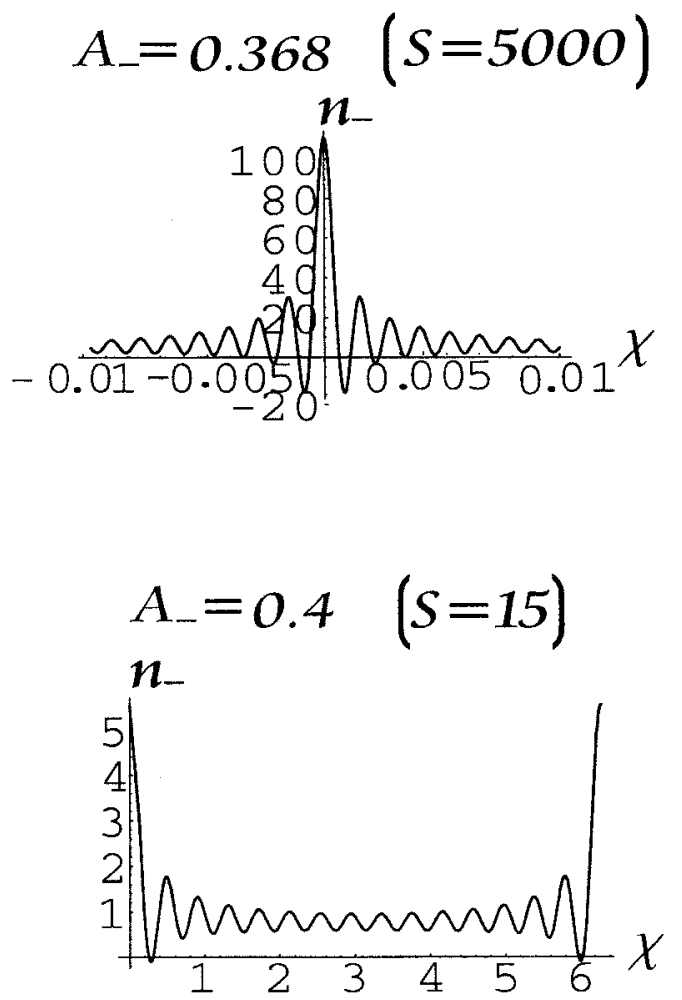

Fig. 1. Graphs of $\chi(=\omega t+\boldsymbol{k} \cdot \boldsymbol{r})$ against $n_{-}$(particle density) for various values of the amplitude $A_{-}$and $S$ (the number of terms taken into account) with $n_{0}$ (the particle density at equilibrium) being scaled to one. For divergent series (i.e., when the series involves $A_{-}$greater or approximately equal to $\mathrm{e}^{-1}$ ) the graphs give some negative values in $n_{-}$, which is physically prohibited. But for the convergent series (e.g., when $A_{-}=0.3$ or any value less than $\mathrm{e}^{-1}$ ), all values of $n_{-}$(in the graphs) are always positive (based on the numerical calculations up to order 7000). Once the limit of convergence is exceeded divergency occurs using few terms: for the limiting case $\left(A_{-}=0.368\right)$ one needs a lot of terms to have a negative $n_{-}$(illustrated by $\left.S=5000\right)$, while for $A_{-}=0.37$ three hundred terms are largely sufficient and for $A_{-}=0.4$ fifteen terms are sufficient. This suggests a method to determine the convergence limits in Table IV. 


\subsubsection{Energy}

Here we consider the convergence of the series for potential energy density $\left(p e=e\left(n_{0}-n_{-}\right) \varphi\right)$, kinetic energy density $\left(k e=0.5 m_{-} n_{-} v_{-}^{2}\right)$, energy density (i.e., $\left.p e+k e\right)$, $\int_{0}^{2 \pi}(p e \times \overline{p e}) \mathrm{d} \chi, \int_{0}^{2 \pi}(k e \times \overline{k e}) \mathrm{d} \chi$ and $\int_{0}^{2 \pi}($ energy $\times \overline{\text { energy }})$ $\mathrm{d} \chi$, where $\overline{p e}, \overline{k e}$ and $\overline{e n e r g y}$ are the complex conjugates. Each of these series converges with $\left|A_{-}\right|<1 /$ e. One can generally show this by considering two series $a=a_{1} A_{-}+a_{2} A_{-}^{2}+a_{3} A_{-}^{3}+\cdots+a_{n} A_{-}^{n}+\ldots$ and $b=$ $b_{1} A_{-}+b_{2} A_{-}^{2}+b_{3} A_{-}^{3}+\cdots+b_{n} A_{-}^{n}+\cdots$ which have properties that $a_{n+1} / a_{n}=e \pm \kappa_{n}$ and $b_{n+1} / b_{n}=e \pm \tau_{n}$ respectively. Here $\kappa_{n}$ and $\tau_{n}$ are small quantities converging to zero when $n \rightarrow \infty$. From $a_{n+1} / a_{n}=e \pm \kappa_{n}$ one gets $a_{2}=a_{1}\left(e \pm \kappa_{1}\right), a_{3}=a_{2}\left(e \pm \kappa_{2}\right)=a_{1}\left(e \pm \kappa_{1}\right)\left(e \pm \kappa_{2}\right)$ and so on. Similarly for $b_{n+1}$. We then take the product of the series $a$ and $b$. From the product, we divide the term involving $A_{-}^{3}$ by that involving $A_{-}^{2}$ to get

$A_{-}[2 e \pm$ (peanuts)].

If we divide terms involving $A_{-}^{4}$ by the one involving $A_{-}^{3}$ we obtain

$A_{-}\left(\frac{3 e^{2} \pm e \text { (peanuts) }}{2 e \pm \text { (peanuts) }}\right)$

Again dividing terms involving $A_{-}^{n}$ by those containing $A_{-}^{n-1}$ we get

$A_{-}\left(\frac{(n-1) e^{n-2} \pm e(\text { peanuts })}{(n-2) e^{n-3} \pm e(\text { peanuts })}\right)$

In the limit this gives $e A_{-}$which implies that, for our series to converge it is required that $\left|A_{-}\right|<1 / e$, just like the cases considered previously.

For visualization purposes, we calculated pe, ke and energy for some $A_{-}$when $\chi=0$. We summarize the result in Table II. From the table we see that the virial theorem $(2 k e=p e)$ is approximately satisfied especially when $A_{-} \leq 0.1$ as it should as the deviation from equilibrium is smaller when $A_{-}$is small. With this result and the convergence of the series considered before, we conclude here that the expressions for the particle density, velocity and electrical potential verify the energy conservation law.

\subsubsection{Discussion}

- In Section 3.1 we considered three procedures, counting Eqs. (17) and (18) as belonging to the same procedure, in

Table II. Table showing various values of energy densities (in units $\mathrm{m}_{-} \mathrm{n}_{0} \omega^{2} \mathrm{~A}_{-}^{2} / \mathrm{k}^{2}$ ) for various values of $\mathrm{A}_{-}$. The energies increase steadily with the amplitude, and approach infinity when $\mathrm{A}_{-}$approaches $e^{-1}$. (Cold plasma case.)

\begin{tabular}{llll}
\hline$A_{-}$ & Potential energy & Kinetic energy & Total energy \\
\hline 0.001 & 1.0025 & 0.5015 & 1.5040 \\
0.01 & 1.0256 & 0.5154 & 1.5410 \\
0.1 & 1.3294 & 0.704 & 2.0335 \\
0.2 & 1.9730 & 1.13335 & 3.1063 \\
0.25 & 2.6122 & 1.5903 & 4.2024 \\
0.3 & 3.9366 & 2.6060 & 6.5426 \\
\hline
\end{tabular}

order to complete the reduction process of our system. Of the three procedures one notes that procedure 2 is shorter, simpler and safer than the other two. This means that a second order equation is easier to handle than an equivalent first order one or a fully integrated one. To some extent we may understand this because a higher order differential equation is usually more condensed than any of its integrated forms. Nevertheless it is surprising as one might have hoped that a fully integrated expression would yield quickly the required series. However it is a lucky aspect as for more complicated equations it may be very difficult to obtain integrated forms.

- We see in our analysis that, the series yields results and converges but only for amplitudes which involve less than $37 \%$ of the equilibrium density. This is seriously different, but not too drastic, from the case of incompressible media treated in [1] where the method was well suited up to large amplitudes and agreed with experiments. However, in the case in [1] the coefficients depended on $k$ and for $k \rightarrow 0$ the coefficients became very large.

- A sketch of $(\omega, k)$ graph for equation (23) is a straight line passing through $\left(0, \omega_{-}\right)$and parallel to $k$. This means $\omega$ does not depend on $k$. Hence the group velocity $\partial \omega / \partial k$ is zero, implying that cold plasma oscillations are not propagating as is well known. This is so because we neglected the kinetic motions of the charged particles.

- From the graphical results of Section 3.1.2 we noted that, there is an agreement between the results and the issues of convergence and divergence. This coincidence encourages us therefore to use the graphical procedure in approximating the radius of convergence in the situations where it is difficult to determine such a value analytically.

- It is suggested that the convergence should be checked experimentally: e.g., by inducing externally applied waves with large amplitudes in the plasma.

\subsubsection{Analysis of perturbation using cosines}

Here we address the question: what happens if we use cosines (or sines) instead of exponentials?

In Section 3.1.1 we noted that using Eq. (20), one gets results much faster than when one uses Eqs. (17), (18), or (24). We therefore, use (20) in the determination of various coefficients when (26) is replaced by

$$
\begin{aligned}
n_{-} & =n_{0}+n_{-1}+n_{-2}+\cdots \\
& =n_{0}+\sum_{i=1}^{S+1} \sum_{s=i}^{S} n_{0} a_{-s, i \rho} A_{-}^{s} \cos (\mathrm{i} \chi),
\end{aligned}
$$

where $S$ goes to infinity and $a_{-s, i \rho}$ are constants to be determined. With the help of the mathematica program we obtained results given in Table III. The first value in Table III (i.e., $a_{-1,1 \rho}$ ) must be different from zero otherwise we will have no perturbation. We therefore put $a_{-1,1 \rho}=1$ and from the linearized case one obtains (23). Proceeding with calculations involving higher orders, we found that all $a_{-s, 1 \rho}$ 's are arbitrary constants. Hence they are not linked to the first order term under consideration and we have to put all $a_{-s, 1 \rho}$ 's (where $s=2,3, \ldots$ ) equal to zero. 
Table III. Table showing the coefficients of $\mathrm{A}^{\mathrm{s}} \cos (l \chi)$ in the series for (a) electron density (i.e., $\mathrm{a}_{-\mathrm{s}, 1 \rho}$ ) with 1 and $\mathrm{s}$ taking values from $1-5,(b)$ velocity (i.e., $\mathrm{a}_{-\mathrm{s}, \mathrm{lv}}$ in units $\omega / \mathrm{k}$ ) and $(c)$ potential (i.e., $\mathrm{a}_{-\mathrm{s}, 1 \varphi}$ in units en $\left.n_{0} / k^{2} \varepsilon\right)$. (Cold plasma case.)

\begin{tabular}{|c|c|c|c|c|c|}
\hline$s$ & 1 & 2 & 3 & 4 & 5 \\
\hline$a_{-s, 1 \rho}$ & 1 & 0 & 0 & 0 & 0 \\
\hline$a_{-s, 2 \rho}$ & & 1 & 0 & $\frac{-1}{12}$ & 0 \\
\hline$a_{-s, 3 \rho}$ & & & $\frac{9}{8}$ & 0 & $\frac{-27}{128}$ \\
\hline$a_{-s, 4 \rho}$ & & & & $\frac{4}{3}$ & 0 \\
\hline$a_{-s, 5 \rho}$ & & & & & $\frac{625}{384}$ \\
\hline$a_{-s, 1 v}$ & -1 & 0 & $\frac{3}{4}$ & 0 & $\frac{-17}{96}$ \\
\hline$a_{-s, 2 v}$ & & $\frac{-1}{2}$ & 0 & $\frac{11}{24}$ & 0 \\
\hline$a_{-s, 3 v}$ & & & $\frac{-3}{8}$ & 0 & $\frac{51}{128}$ \\
\hline$a_{-s, 4 v}$ & & & & $\frac{-1}{3}$ & 0 \\
\hline$a_{-s, 5 v}$ & & & & 0 & $\frac{-125}{384}$ \\
\hline$a_{-s, 1 \varphi}$ & -1 & 0 & $\frac{3}{2}$ & 0 & $\frac{-15}{8}$ \\
\hline$a_{-s, 2 \varphi}$ & & $\frac{-1}{4}$ & 0 & $\frac{19}{48}$ & 0 \\
\hline$a_{-s, 3 \varphi}$ & & & $\frac{-1}{8}$ & 0 & $\frac{27}{128}$ \\
\hline$a_{-s, 4 \varphi}$ & & & & $\frac{-1}{12}$ & 0 \\
\hline$a_{-s, 5 \varphi}$ & & & & & $\frac{-25}{384}$ \\
\hline
\end{tabular}

The results in Table III were then compared with results given in Table I. Doing so one notes that the coefficients $a_{-s, s f}$ (with $f$ here standing for either $\rho, v$ or $\varphi$ ) are related to $a_{-s f}$ through a formula

$a_{-s, s f}=\frac{a_{-s f}}{2^{s-1}}$.

For other values (non-diagonal ones) in Table III, one may infer some general formulae for example $a_{-s, s-2 f}=$ $-\left[(s-2)^{s-1}(s-3) !\right] /\left[2^{s}(s-1) !(s-4) !\right]$, with $s=4,5 \cdots$.

Checking for convergence of (36) with (37) (putting $f=\rho$ ), one finds that the series converges with $\left|A_{-}\right|<2 / \mathrm{e}$. The same applies when $f$ is replaced by $v$ or $\varphi$. In fact $\mathrm{e}^{s i \chi}=\cos s \chi+\mathrm{i} \sin s \chi$ consists physically of a sum of two waves. Using only one (say the cosine, for example) reduces the wave and its energy by a factor two, thus allowing the amplitude to be doubled. As in experiments one deals rather with a sinusoidal wave and not an exponential one, we have $2 / \mathrm{e}$ as the practical limit of convergence for $|A|$. This amounts to amplitudes up to $72 \%$ of the initial density. Experimental verification is solicited.

\subsection{Sound waves}

Sound waves are described here by

$\partial_{t} \rho+\operatorname{div}(\rho v)=0$

$\rho \frac{\mathrm{d} v}{\mathrm{~d} t}=-\nabla p$

$p=K \rho^{\Gamma}$,

where $\rho$ is the density of the medium. As in Section 3.1, all quantities are taken here to be functions of $\chi$ alone. Hence the above system is reduced to

$\left(\omega^{2} \rho_{0}^{2}-k^{2} \Gamma K \rho^{\Gamma+1}\right) \rho^{\prime}=0$

which implies that either

$\rho^{\prime}=0(\Rightarrow \rho$ is a constant $)$

or

$\omega^{2} \rho_{0}^{2}=k^{2} \Gamma K \rho^{\Gamma+1}$.

Expression (42) would require that our system is incompressible, prohibiting sound waves (i.e., requiring the amplitude to be vanishingly small). Equation (43) (which yields the dispersion relation for sound waves when $\rho=\rho_{0}$ is substituted) requires $\rho$ to be constant too, unless $\Gamma=-1$. The case $\Gamma=-1$ has no physical sense but when we use it in the Eqs. (38)-(40) it turns out that $\rho$ again has to be constant. Clearly in all cases the radius of convergence is zero. For more insight we analyze Eqs. (38)(40) after substituting various orders.

\subsubsection{Attempt with series development}

In this part we consider again Eqs. (38)-(40) and analyze them after substituting quantities from various orders.

First order

Clearly $\rho_{0}$ and $p_{0}$ are constant with respect to space and time. Hence putting $\rho=\rho_{0}+\rho_{1}, p=p_{0}+p_{1}$ and $\boldsymbol{v}=\boldsymbol{v}_{1}$ into the system (38)-(40) and simplifying we get

$\partial_{t} \rho_{1}=-\rho_{0} \boldsymbol{\nabla} \cdot \boldsymbol{v}_{1}$,

$\rho_{0} \partial_{t} \boldsymbol{v}_{1}=-v_{s}^{2} \nabla \rho_{1}$.

Taking the divergence on (45) we have

$\rho_{0} \partial_{t}\left(\nabla \cdot \boldsymbol{v}_{1}\right)=-v_{s}^{2} \Delta \rho_{1}$,

since $\partial_{t}$ and $\boldsymbol{\nabla}$ can commute. Substituting (44) into (46) we get

$\left(\partial_{t t}^{2}-v_{s}^{2} \Delta\right) \rho_{1}=0$.

We then let

$\rho=\rho_{0}+\rho_{1}+\rho_{2}+\cdots=\sum_{s} \rho_{0} \alpha_{s} A^{s} \mathrm{e}^{\mathrm{i} s \chi}$, 
where $\alpha_{0}=\alpha_{1}=1$. Substituting this into (47) leads to the dispersion relation for sound waves:

$$
\omega^{2}=k^{2} \Gamma K \rho_{0}^{\Gamma-1} \equiv k^{2} v_{s}^{2} .
$$

For $p_{1}$ and $\boldsymbol{v}_{1}$ we find

$p_{1}=v_{s}^{2} \rho_{1}=v_{s}^{2} A \rho_{0} \mathrm{e}^{\mathrm{i} \chi}$

and

$\boldsymbol{v}_{1}=-\boldsymbol{k} \omega A \mathrm{e}^{\mathrm{i} \chi} / k^{2}=-\boldsymbol{k} v_{s}^{2} \rho_{1} /\left(\omega \rho_{0}\right)$.

\section{Second order}

Expanding and neglecting all quantities involving orders higher than two and eliminating $\boldsymbol{v}_{2}$ yields

$$
\begin{gathered}
\left(\partial_{t t}^{2}-v_{s}^{2} \Delta\right) \rho_{2}=-\left[\left(\partial_{t} \rho_{1}\right)\left(\boldsymbol{\nabla} \cdot \boldsymbol{v}_{1}\right)+\rho_{1} \partial_{t}\left(\boldsymbol{\nabla} \cdot \boldsymbol{v}_{1}\right)\right. \\
\left.+\left(\partial_{t} \boldsymbol{v}_{1}\right)\left(\boldsymbol{\nabla} \rho_{1}\right)+\boldsymbol{v}_{1} \cdot \nabla\left(\partial_{t} \rho_{1}\right)\right]+\rho_{0} \nabla\left(\boldsymbol{v}_{1} \cdot \nabla \boldsymbol{v}_{1}\right) \\
+\left(\nabla \rho_{1}\right) \partial_{t} \boldsymbol{v}_{1}+\rho_{1} \boldsymbol{\nabla} \partial_{t} \boldsymbol{v}_{1}+\frac{v_{s}^{2}(\Gamma-1)}{2 \rho_{0}} \Delta \rho_{1}^{2} .
\end{gathered}
$$

Putting (51) into (52) shows that $\rho_{2}$ has to be of the form $\alpha_{2} \rho_{1}^{2} / \rho_{0}$ with $\alpha_{2}$ a dimensionless constant. This yields

$\alpha_{2} \rho_{1}^{2}\left(\omega^{2}-k^{2} v_{s}^{2}\right)=\rho_{1}^{2}\left[\omega^{2}+k^{2} v_{s}^{2}(\Gamma-1) / 2\right]$.

Since $\omega^{2}-v_{s}^{2} k^{2}$ is zero we must have $\Gamma=-1$, which is physically meaningless, or $\alpha_{2}$ has to be infinite, which just means that the series diverges for any amplitude. Mathematically the problem may be avoided by adding a factor $\chi$ (or $t$ or $\boldsymbol{r}$ ) in the expression for $\rho_{2}$, but this leads to diverging amplitudes and has physically a very limited value.

From the analysis above it is clear that, for pure sound waves (no electromagnetic interaction) the series either diverges (meaning that instability has to occur or one of the assumptions is wrong) or gives results which are physically meaningless. However sound waves do exist. This motivates us to investigate further the sound wave problem in question. We therefore generalize the above approach so as to have more insight about our method and the problem itself.

\subsubsection{A more general approach}

Eliminating $p$ between (39) and (40) yields

$\partial_{t} \boldsymbol{v}+(\boldsymbol{v} \cdot \nabla) \boldsymbol{v}=-K \Gamma \rho^{\Gamma-2} \nabla \rho$.

Let $\rho=\rho_{0}+\varepsilon \rho_{1}+\varepsilon^{2} \rho_{2}+\varepsilon^{3} \rho_{3}+\cdots$ and $\boldsymbol{v}=\varepsilon \boldsymbol{v}_{1}+\varepsilon^{2} \boldsymbol{v}_{2}+$ $\varepsilon^{3} \boldsymbol{v}_{3}+\cdots$, where $\varepsilon$ is now a small arbitrary constant.

\section{First order}

For the first order we have

$\partial_{t} \rho_{1}+\operatorname{div}\left(\rho_{0} \boldsymbol{v}_{1}\right)=0$

and

$\partial_{t} \boldsymbol{v}_{1}=-K \Gamma \rho_{0}^{\Gamma-2} \nabla \rho_{1}$.
Elimination of $\boldsymbol{v}_{1}$ yields the wave equation given in (47). This has a general solution which can be written as the sum of eight terms of the type

$\rho_{1}=f(\chi)$,

with $\omega / k=v_{s}$ by definition and $f$ an arbitrary function. Substituting this into (55) yields

$\partial_{t} \boldsymbol{v}_{1}=-v_{s}^{2} \boldsymbol{k} f^{\prime} / \rho_{0}$.

Integrating (56) with respect to $t$ we get

$\boldsymbol{v}_{1}=-v_{s}^{2} \boldsymbol{k} f /\left(\omega \rho_{0}\right)+C(\boldsymbol{r})$.

We may put $C(\boldsymbol{r})=\mathbf{0}$ since for vanishing $f$ we must have vanishing $\boldsymbol{v}_{1}$.

Second order

Here we obtain upon substituting (54) and (55):

$\partial_{t} \rho_{2}+\rho_{0} \boldsymbol{\nabla} \boldsymbol{v}_{2}+\rho_{1} \boldsymbol{\nabla} \boldsymbol{v}_{1}+\boldsymbol{v}_{1} \nabla \rho_{1}=0$

and

$\partial_{t} \boldsymbol{v}_{2}+\boldsymbol{v}_{1} \cdot \boldsymbol{\nabla} \boldsymbol{v}_{1}=-\frac{v_{s}^{2}}{\rho_{0}}\left[\boldsymbol{\nabla} \rho_{2}+\frac{(\Gamma-2) \rho_{1} \nabla \rho_{1}}{\rho_{0}}\right]$

Taking the divergence of (59) and substituting (58) into the new expression in order to get rid of $\boldsymbol{v}_{2}$ yielding

$$
\begin{aligned}
& \left(\partial_{t t}^{2}-v_{s}^{2} \Delta\right) \rho_{2}=\frac{v_{s}^{2}(\Gamma-2)\left(\nabla \rho_{1} \cdot \nabla \rho_{1}+\rho_{1} \Delta \rho_{1}\right)}{\rho_{0}} \\
& \quad+\rho_{0}\left(\nabla \boldsymbol{v}_{1} \cdot \nabla \boldsymbol{v}_{1}+\boldsymbol{v}_{1} \Delta \boldsymbol{v}_{1}\right)-\partial_{t}\left(\rho_{1} \nabla \boldsymbol{v}_{1}+\boldsymbol{v}_{1} \nabla \rho_{1}\right) .
\end{aligned}
$$

For illustration purposes, we consider the following two cases

(a) Let $f=\mathrm{e}^{\mathrm{i} \chi}$. Putting this into (57) and then combining the obtained equation with (60) to have

$\left(\partial_{t t}^{2}-v_{s}^{2} \Delta\right) \rho_{2}=C_{h} f^{2}$

where $C_{h}=-\left[2 k^{2} v_{s}^{2}(\Gamma+1)\right] / \rho_{0}$. In a general way the solution of a differential equation of this type (i.e., with differentials on the left-hand side and a given function on the right-hand side) consists of the sum of two parts: one part is a solution of the equation without the right-hand side (omitting the given function), the other part is a particular solution with the right-hand side (note: any particular solution will do!). The general solution of the left-hand side is (restricting $\boldsymbol{k} \cdot \boldsymbol{r}$ to $k x$ )

$f_{+}(\omega t+k x)+f_{-}(\omega t-k x)$

with $f_{+}$and $f_{-}$arbitrary functions of their argument. However the solution without right-hand side has nothing to do with the first order solution under consideration, so we drop it. For a particular solution with right-hand side one has usually to take a function like the one on the right-hand side. However here $f$ on the right-hand side satisfies the left-hand side (this is called "absorption") and one has to adapt a function 
with a supplementary factor. Hence we find that

$\rho_{2}=C_{h} t \mathrm{e}^{2 \mathrm{i}(\omega t+k x)} /(4 \mathrm{i} \omega)$

is a particular solution (a similar solution with $x$ in front of the exponential is a particular solution too: the difference between both particular solutions belongs to the solutions of the left-hand side.) This is the relevant solution for our nonlinear analysis. However it is no more periodic. We may construct higher order terms (involving higher powers of $t$ or $x$ ), but clearly the solution will have limited application: for large $t$ the oscillations become very wild. To avoid this one often uses an adapted $\omega /$ which is shifted somewhat from $\omega$, for the second order term. However, it is clear that this again means that the solution is not really periodic although the first order term is periodic. In either of those procedures the convergence or the range of application is not clear, cf. the introduction.

(b) Let $f=(\omega t+k x)^{s}$, where $s=1,2, \ldots$ Using this in (60) gives

$\left(\partial_{t t}^{2}-v_{s}^{2} \Delta\right) \rho_{2}=C_{q} f^{2(1+1 / s)}$.

where $C_{q}=k^{2} v_{s}^{2}(\Gamma+1) s(2 s-1) / \rho_{0}$. In a similar way as for case (a), we find the particular solution:

$\rho_{2}=C_{q} t(\omega t+k x)^{3+2 / s} /(2 \omega(3+2 / s))$.

The solution is no more dependent on $\chi$ alone but separately on $t$ as well. Again in higher order terms powers of $t$ (or $x$ ) may occur. However the situation is quite different from the previous one: indeed here the first order solution was not a periodic function, rather a kind of traveling wave. However the nonlinear terms alter this character.

In our analysis of sound waves the phase velocity is constant and the dispersion relation gives a straight line through the origin. Hence $\omega / k=n \omega / n k$ for all $(\omega, k), n$ arbitrary. This makes $\left(\partial_{t t}^{2}-v_{s}^{2} \Delta\right)=0$ for all $(\omega, k)$ and all $n$. However, the right-hand side, although differing from case to case depending on the choice of $f$, is different from zero and thus would require infinite coefficients on the left-hand side.

\subsection{Plasma with pressure of electrons}

Here we assume that the kinetic motion of electrons is not negligible and thus the pressure of electrons is present. Hence our basic system of equations becomes Eqs. (6), (8) together with

$n_{-} m_{-} \frac{\mathrm{d} \boldsymbol{v}_{-}}{\mathrm{d} t}=-\nabla p_{-}+e n_{-} \nabla \varphi$

and

$p_{-}=K_{-} n_{-}^{\Gamma_{-}}$.

\subsubsection{Elimination}

We once more let all quantities be functions of $\chi$ alone. Hence as we did previously in Section 3.1, we reduce the above system to

$\varphi^{\prime}=\frac{m_{-}\left(k^{2} v_{s-}^{2} n_{-}^{\Gamma_{-}+1}-\omega^{2} n_{0}^{\Gamma_{-}+1}\right) n_{-}}{e n_{-}^{3} k^{2} n_{0}^{\Gamma_{-}-1}}$,

where $v_{s-}^{2}=K_{-} \Gamma_{-} n_{0}^{\Gamma_{-}-1} / m_{-}$is the sound velocity of electrons. Equation (63) is the same as (15) with the exception of the pressure term added. Hence we complete the reduction process as follows:

Procedure 1: (Reduction to nonlinear differential equation of first order).

Here we repeat procedure 1 of Section 3.1, using however $\varphi$ ' from (63) to obtain

$$
\begin{aligned}
& \left(\frac{k^{2} v_{s-}^{2} n_{-}^{\Gamma_{-}+1}}{n_{0}^{\Gamma_{-}-1}}-\omega^{2} n_{0}^{2}\right)^{2} n^{\prime 2}+\omega^{2} \omega_{-}^{2} n_{-}^{4}\left(n_{-}-n_{0}\right)^{2} \\
& =\frac{2 \omega_{-}^{2} v_{s-}^{2}\left[\Gamma_{-} n_{-}^{\Gamma_{-}-1}\left(n_{-}-n_{0}\right)-\left(n_{-}^{\Gamma_{-}}-n_{0}^{\Gamma_{-}}\right)\right]}{\Gamma_{-}\left(\Gamma_{-}-1\right) n_{0}^{\Gamma_{-}} k^{-2} n_{-}^{-6}} .
\end{aligned}
$$

This is the equation to be used in one of the mathematica programs in order to determine $b_{-s \rho}$ 's as we shall see later. For the potential we first integrate (63) with respect to $\chi$ to get

$\varphi+C=\frac{m_{-} n_{0}}{e k^{2} n_{-}^{2}}\left[\frac{k^{2} v_{s-}^{2} n_{-}^{\Gamma_{-}+1}}{\left(\Gamma_{-}-1\right) n_{0}^{\Gamma_{-}}}+\frac{\omega^{2} n_{0}}{2}\right]$.

We then determine $C$ by substituting $n_{-}=n_{0}$ and $\varphi_{0}=0$ into (65). Hence

$\varphi=\frac{m_{-}}{e k^{2}}\left[\frac{v_{s-}^{2}\left(n_{-}^{\Gamma_{-}-1}-n_{0}^{\Gamma_{-}-1}\right)}{k^{-2}\left(\Gamma_{-}-1\right) n_{0}^{\Gamma_{-}-1}}+\frac{\omega^{2}}{2 n_{-}^{2}}\left(n_{0}^{2}-n_{-}^{2}\right)\right]$.

Procedure 2: (Reduction to nonlinear differential equation of second order).

Differentiate (63) with respect to $\chi$ and substitute into (11) to get

$$
\begin{aligned}
& \left(\frac{k^{2} v_{s-}^{2}\left(\Gamma_{-}-2\right) n_{-}^{\Gamma_{-}+1}}{n_{0}^{\Gamma_{-}}}+3 \omega^{2} n_{0}\right) n_{-}^{\prime 2} \\
& \quad+\left(\frac{k^{2} v_{s-}^{2} n_{-}^{\Gamma_{-}+1}}{n_{0}^{\Gamma_{-}}}-\omega^{2} n_{0}\right) n_{-} n_{-}^{\prime \prime}=\frac{\omega_{-}^{2}\left(n_{-}-n_{0}\right)}{n_{0}^{2} n_{-}^{-4}} .
\end{aligned}
$$

This may be used equally well as (64) in the determination of $n_{-}$. In fact it turns out to be simpler than Eq. (64).

\subsubsection{Dispersion Relation}

Here we let

$n_{-}=n_{0}+n_{-1}+\cdots=\sum_{s=0}^{N} n_{0} b_{-s \rho} A^{s} \mathrm{e}^{\mathrm{i} s \chi}$, 
where $N$ goes to infinity, $b_{-0 \rho}=1=b_{-1 \rho}$ and the remaining coefficients, $b_{-s \rho}$, are to be determined. Hence, putting (68) into (67) and linearizing yields

$$
\omega^{2}=\omega_{-}^{2}+k^{2} v_{s-}^{2}
$$

which is the (Langmuir) dispersion relation for electron plasma waves [9, p.146]. Note that Eq. (69) is slightly more general than the usual Langmuir equation as it uses $\Gamma_{-}$ instead of $\gamma_{-}$, the ratio of specific heats.

Solving (64) with (68) having $N=2$, we obtain (69) together with

$\omega^{2}=k^{2} v_{s-}^{2}$.

We reject this since it contradicts (69) (cf. Section 3.1.1).

\subsubsection{Analysis of perturbation when exponentials are used}

We solved Eq. (67) (with $\omega^{2}$ being replaced by (69)) up to order fifteen and determined $b_{-s \rho}$ 's. Similarly we evaluated the constants $b_{-s v}$ and $b_{-s \varphi}$ for the velocity and potential. See appendix I for the first five coefficients. Identical results are obtained if one solves (64), however with more effort.

\section{Comments on the convergence}

Figure 2 gives $(\omega, k)$ graphs for the dispersion relations for cold plasma and for plasma with electron pressure together with the oblique asymptote (dispersion relation for sound waves). Let $k_{m}$ be the value of the wavenumber at the point of intersection of graphs for Eqs. (23) and that of the oblique asymptote. If $k \ll k_{m}$ (long wavelengths), then electric forces dominate and the plasma frequency $\omega_{-}$of the electrons is a fair approximation. This is due to the fact that the electrostatic potential has a long range and hence becomes relatively more important for longer wavelengths than the pressure variations. When $k \gg k_{m}$ (short wavelengths), then the approximation for the sound waves dominated and the convergence decreases. If $k=k_{m}$, then we have the wavenumber at the approximated point of transition between the cold plasma region and the sound

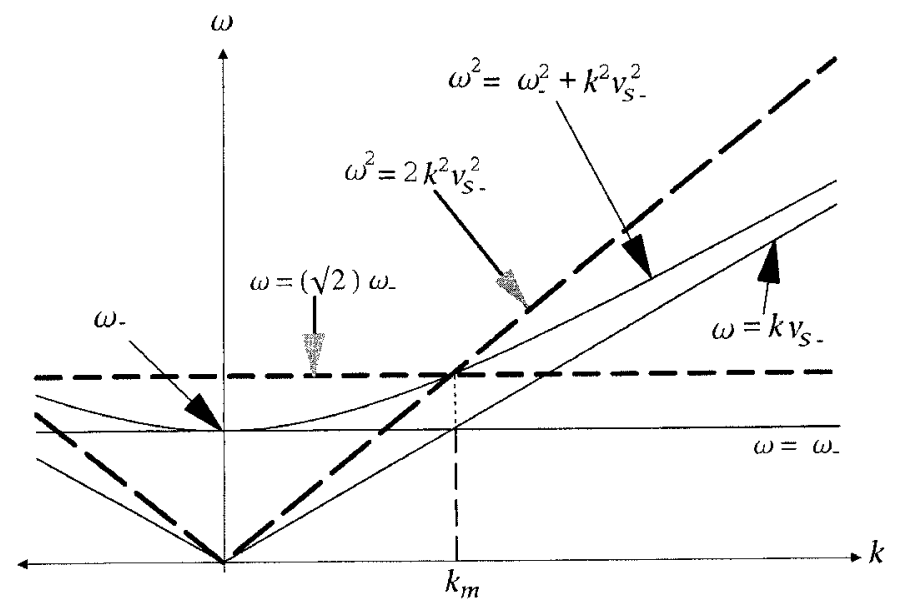

Fig. 2. $(\omega, k)$ graphs for the dispersion relations for cold plasma, sound waves and plasma with electron pressure. (We sketched mainly one branch because $\pm k$ are not really distinct). The straight lines passing through the origin correspond to pure sound waves and are the asymptotes of a hyperbola. For comparison purposes, this figure gives also the graphs of $\omega^{2}=2 \omega_{-}^{2}$ and $\omega^{2}=2 k^{2} v_{s-}^{2}$, which were obtained when an electronpositron plasma case was considered. dominated region. The expression for $k_{m}$ can be determined as follows:

First put $p_{-0}=n_{0} k_{B} T_{-}$. Hence

$k_{m}=\frac{\omega_{-}}{v_{s-}}=e \sqrt{\frac{n_{0}}{\varepsilon \Gamma_{-} k_{B} T_{-}}}$.

From our results in Appendix I we note that the coefficients of the higher order terms are polynomials in the parameter $\omega_{\Omega}=\left(1+\Gamma_{-}\right) k^{2} v_{S_{-}}^{2} / \omega_{-}^{2}=k^{2} \Gamma_{-}(1+$ $\left.\Gamma_{-}\right) \Lambda_{D-}^{2}$, where $\Lambda_{D-}^{2}=\varepsilon K_{-} n_{0}^{\Gamma_{-}} /\left(e n_{0}\right)^{2}$. (Note that, if $\Gamma_{-}=\gamma_{-}$then $\Lambda_{D-}^{2}=\lambda_{D-}^{2}$, which is the square of the Debye length). The parameter $\omega_{\Omega}$ is a measure of the relative importance of the pressure and the electric action. Moreover the convergence depends now on $k$, in contrast to the cold plasma case. Substituting Eq. (71) into this parameter, we obtain

$\omega_{\Omega}=\left(\Gamma_{-}+1\right)\left(k / k_{m}\right)^{2}$.

To fix the ideas, we take $\Gamma_{-}=\gamma_{-}=5 / 3$ (monoatomic). For $\omega_{\Omega}=1 / 2$ (i.e., $k=\sqrt{3} k_{m} / 4$ ) we find graphically that $\left|A_{-}\right|<0.775 \mathrm{e}^{-1}$ for convergence (based on the terms up to fifteenth order). This may be compared with $\left|A_{-}\right|<\mathrm{e}^{-1}$ for cold plasma $\left(\omega_{\Omega}=0\right)$. Other results for the cases $k \ll k_{m}, k=k_{m}$ and $k \gg k_{m}$ are summarized in Table IV. Hence inclusion of the electron motion makes the convergence slower and the domain of convergence smaller. The more the $\omega, k$ curve approaches the straight line asymptote of sound waves, the worse the convergence.

Again experimental verifications using externally applied waves of various frequency are proposed.

It may be noted that if the incompressibility increases (thus $\Gamma_{-}$increases) $\omega_{\Omega}$ increases and thus the convergence decreases. This is physically understandable as the pressure increases (relative to the electric forces) the convergence decreases. This is in agreement with the fact that cold plasma waves have a fair convergence while sound waves have no convergence at all.

\subsubsection{Analysis of perturbation when cosines are used}

Here we substitute Eq. (36) (with " $a$ " being replaced by " $b$ ") into Eq. (67) and solve for the required coefficients

Table IV. Some values of the maximum allowed $\left|\mathrm{A}_{-}\right|$to have convergence in various cases of $\mathrm{k}$, where $\omega_{\Omega}=\left(1+\Gamma_{-}\right)$ $\mathrm{k}^{2} \mathrm{v}_{\mathrm{s}-}^{2} / \omega_{-}^{2}$ and $\mathrm{k}_{\mathrm{m}}=\omega_{-} / \mathrm{v}_{\mathrm{s}-}$. (Plasma with electron pressure.)

\begin{tabular}{llll}
\hline & $\omega_{\Omega}$ & $k$ & $\left|A_{-}\right|$ \\
\hline & 0 & $0\left(v_{s-}=0\right.$ or $\left.k_{m}=\infty\right)$ & $<\mathrm{e}^{-1}$ \\
$k \ll k_{m}$ & $\frac{1}{2}$ & $\frac{\sqrt{3}}{4} k_{m}$ & $<0.775 \mathrm{e}^{-1}$ \\
& 1 & $\frac{\sqrt{3}}{2 \sqrt{2}} k_{m}$ & $<0.612 \mathrm{e}^{-1}$ \\
$k=k_{m}$ & $\frac{8}{3}$ & $k_{m}$ & $<0.353 \mathrm{e}^{-1}$ \\
& 8 & $\sqrt{3} k_{m}$ & $<0.163 \mathrm{e}^{-1}$ \\
$k \gg k_{m}$ & 800 & $10 \sqrt{3} k_{m}$ & $<0.0019 \mathrm{e}^{-1}$ \\
& 8000 & $10 \sqrt{30} k_{m}$ & $<0.00019 \mathrm{e}^{-1}$ \\
\hline
\end{tabular}


with the assistance of mathematica up to order seven. Comparing these values with those of Appendix I.1 we infer the formula

$b_{-s, s \rho}=b_{-s \rho} / 2^{s-1}$.

It is again possible to infer general formulae for the remaining values as well as for those in velocity and potential. Due to the factor $2^{s-1}$ in the denominator the convergence is granted up to amplitudes twice as large as in the case of exponentials. In fact exponentials correspond to two waves (or doubling the amplitude). However, in experiments one has to think in terms of sinusoidal waves. Cf. Section 3.1.5.

\section{Plasma with perturbed ion density}

We consider the case in which both ions and electrons may move. Hence our basic system of equations here is made up of Eqs. (1)-(5).

\subsection{Approach I: Reduction to nonlinear differential equations of first order}

Following the procedure illustrated in the previous sections, we put our basic system of equations into the following form:

$\left(\omega+\boldsymbol{k} \cdot \boldsymbol{v}_{ \pm}\right) n_{ \pm}^{\prime}+n_{ \pm} \boldsymbol{k} \cdot \boldsymbol{v}_{ \pm}^{\prime}=0$

$m_{ \pm} n_{ \pm}\left(\omega+\boldsymbol{v}_{ \pm} \cdot \boldsymbol{k}\right) \boldsymbol{v}_{ \pm}^{\prime}=-\boldsymbol{k} p^{\prime} \mp e n_{ \pm} \boldsymbol{k} \varphi^{\prime}$

$k^{2} \varphi^{\prime \prime}=e\left(n_{-}-n_{+}\right) / \varepsilon$,

$p_{ \pm}=K_{ \pm} n_{ \pm}^{\Gamma_{ \pm}}$

$p=p_{+}+p_{-}$.

We then reduce our system of equations by eliminating velocities, pressures and potentials as follows:

First integrate (73) to obtain

$\left(\omega+\boldsymbol{k} \cdot \boldsymbol{v}_{ \pm}\right) n_{ \pm}=\epsilon_{ \pm}=\omega n_{0}$.

Then rearrange the equations obtained after substituting (78) into (73) to have

$\boldsymbol{k} \cdot \boldsymbol{v}_{ \pm}^{\prime}=-\omega n_{0} n_{ \pm}^{\prime} / n_{ \pm}^{2}$.

Multiply $\boldsymbol{k}$ on both sides of (74) and substitute (79) to get

$m_{+} \omega^{2} n_{0}^{2} n_{+}^{\prime} / n_{+}^{2}=k^{2}\left(p^{\prime}+e n_{+} \varphi^{\prime}\right)$,

and

$m_{-} \omega^{2} n_{0}^{2} n_{-}^{\prime} / n_{-}^{2}=k^{2}\left(p^{\prime}-e n_{-} \varphi^{\prime}\right)$.

Subtracting (81) from (80) we have

$\omega^{2} n_{0}^{2}\left(\frac{m_{+} n_{+}^{\prime}}{n_{+}^{2}}-\frac{m_{-} n_{-}^{\prime}}{n_{-}^{2}}\right)=e k^{2}\left(n_{+}+n_{-}\right) \varphi^{\prime}$ while summing them (i.e., (81) and (80)), after substituting for $p$, yields

$$
\begin{aligned}
& \frac{\omega^{2} n_{0}^{2}}{k^{2}}\left(\frac{m_{+} n_{+}^{\prime}}{n_{+}^{2}}+\frac{m_{-} n_{-}^{\prime}}{n_{-}^{2}}\right)-e\left(n_{+}-n_{-}\right) \varphi^{\prime} \\
& \quad=2\left(\Gamma_{+} K_{+} n_{+}^{\Gamma_{+}-1} n_{+}^{\prime}+\Gamma_{-} K_{-} n_{-}^{\Gamma_{-}-1} n_{-}^{\prime}\right) .
\end{aligned}
$$

Integrating (75) with respect to $\chi$ and then substituting in (82) and (83) we have respectively

$$
\begin{aligned}
& {\left[\frac{\omega^{2} n_{0}^{2}}{e k^{2}\left(n_{+}+n_{-}\right)}\left(\frac{m_{+} n_{+}^{\prime}}{n_{+}^{2}}-\frac{m_{-} n_{-}^{\prime}}{n_{-}^{2}}\right)\right]^{2}} \\
& \quad=\frac{2 \omega^{2} n_{0}^{2}}{k^{4} \varepsilon} \int \frac{\left(n_{-}-n_{+}\right)}{\left(n_{+}+n_{-}\right)}\left(\frac{m_{+} n_{+}^{\prime}}{n_{+}^{2}}-\frac{m_{-} n_{-}^{\prime}}{n_{-}^{2}}\right) \mathrm{d} \chi .
\end{aligned}
$$

and

$$
\begin{aligned}
& \left\{\frac { 1 } { e ( n _ { + } - n _ { - } ) } \left[\frac{\omega^{2} n_{0}^{2}}{k^{2}}\left(\frac{m_{+} n_{+}^{\prime}}{n_{+}^{2}}+\frac{m_{-} n_{-}^{\prime}}{n_{-}^{2}}\right)\right.\right. \\
& \left.\left.-2\left(\Gamma_{+} K_{+} n_{+}^{\Gamma_{+}-1} n_{+}^{\prime}+\Gamma_{-} K_{-} n_{-}^{\Gamma_{-}-1} n_{-}^{\prime}\right)\right]\right\}^{2} \\
& =-\frac{4}{k^{2} \varepsilon}\left\{\int \left[\frac{\omega^{2} n_{0}^{2}}{2 k^{2}}\left(\frac{m_{+} n_{+}^{\prime}}{n_{+}^{2}}+\frac{m_{-} n_{-}^{\prime}}{n_{-}^{2}}\right)\right.\right. \\
& \left.\left.-\Gamma_{+} K_{+} n_{+}^{\Gamma_{+}-1} n_{+}^{\prime}-\Gamma_{-} K_{-} n_{-}^{\Gamma_{-}-1} n_{-}^{\prime}\right] \mathrm{d} \chi\right\},
\end{aligned}
$$

The integration constants have been put equal to zero as required when we substitute $n_{+}=n_{-}=n_{0}$. Using mathematica program, we solved (84) and (85) with $c_{-0 \rho}=$ $c_{-1 \rho}=1$ and

$n_{ \pm}=n_{0} \sum_{s=0}^{N} c_{ \pm s \rho} A^{s} \mathrm{e}^{\mathrm{i} s \chi}$

where $N$ goes to infinity. In first order we obtain respectively

$c_{+1 \rho}=-\frac{m_{-}}{m_{+}}\left(\frac{\omega^{2}-2 k^{2} v_{s-}^{2}}{\omega^{2}-2 k^{2} v_{s+}^{2}}\right)$

and

$c_{+1 \rho}=\frac{m_{-}}{m_{+}}\left(\frac{\omega^{2}-2 \omega_{-}^{2}}{\omega^{2}-2 \omega_{+}^{2}}\right)$ and $c_{+1 \rho}=\frac{m_{-}}{m_{+}}$

where $v_{s+}^{2}=K_{+} \Gamma_{+} n_{0}^{\Gamma_{+}-1} / m_{+}$and $\omega_{+}^{2}=e^{2} n_{0} /\left(\varepsilon m_{+}\right)$. Equating (87) with (88) we get

$$
\begin{gathered}
\omega^{4}-\omega^{2}\left(\omega_{+}^{2}+\omega_{-}^{2}+k^{2} v_{s-}^{2}+k^{2} v_{s+}^{2}\right) \\
+2 k^{2}\left(v_{s-}^{2} \omega_{+}^{2}+v_{s+}^{2} \omega_{-}^{2}\right)=0
\end{gathered}
$$

and

$\omega^{2}=k^{2}\left(v_{s-}^{2}+v_{s+}^{2}\right)$.

The latter we normally reject (cf. Eqs. (27), (28) and (70) ). However, if electrons and positive particles move together 
this equation may exceptionally be valid (cf. electronpositron plasma below) because it is then included in (89).

\subsection{Approach II: Reduction to nonlinear differential equations of second order}

In the previous approach, $\varphi$ was eliminated from the equation obtained after integrating (75) by the substitution of Eqs. (82) and (83) into it to obtain respectively two equations. Here we eliminate $\varphi$ from (75) by differentiating (82) and (83) with respect to $\chi$ and then substituting the results respectively into Eq. (75) to obtain

$$
\begin{aligned}
\left(n_{+}^{\prime}+\right. & \left.n_{-}^{\prime}\right)\left(-\frac{m_{+} n_{+}^{\prime}}{n_{+}^{2}}+\frac{m_{-} n_{-}^{\prime}}{n_{-}^{2}}\right)+\left(n_{+}+n_{-}\right) \\
& \times\left[m_{+}\left(\frac{n_{+}^{\prime \prime}}{n_{+}^{2}}-\frac{2 n_{+}^{\prime 2}}{n_{+}^{3}}\right)-m_{-}\left(\frac{n_{-}^{\prime \prime}}{n_{-}^{2}}-\frac{2 n_{-}^{\prime 2}}{n_{-}^{3}}\right)\right] \\
= & \frac{e^{2}\left(n_{-}^{2}-n_{+}^{2}\right)\left(n_{+}+n_{-}\right)}{\varepsilon \omega^{2} n_{0}^{2}}
\end{aligned}
$$

and

$$
\begin{aligned}
\omega^{2} n_{0}^{2}\left(n_{-}^{\prime}-n_{+}^{\prime}\right)\left[\left(\frac{m_{+} n_{+}^{\prime}}{n_{+}^{2}}+\frac{m_{-} n_{-}^{\prime}}{n_{-}^{2}}\right)\right. \\
\left.\quad-2 k^{2}\left(\Gamma_{+} K_{+} n_{+}^{\Gamma_{+}-1} n_{+}^{\prime}+\Gamma_{-} K_{-} n_{-}^{\Gamma_{-}-1} n_{-}^{\prime}\right)\right] \\
+\omega^{2} n_{0}^{2}\left(n_{+}-n_{-}\right)\left[m_{+}\left(\frac{n_{+}^{\prime \prime}}{n_{+}^{2}}-\frac{2 n_{+}^{\prime 2}}{n_{+}^{3}}\right)\right. \\
\quad+m_{-}\left(\frac{n_{-}^{\prime \prime}}{n_{-}^{2}}-\frac{2 n_{-}^{\prime 2}}{n_{-}^{3}}\right) \\
\quad-2 k^{2} \Gamma_{+} K_{+}\left(\left(\Gamma_{+}-1\right) n_{+}^{\Gamma_{+}-2} n_{+}^{\prime 2}+n_{+}^{\Gamma_{+}-1} n_{+}^{\prime \prime}\right) \\
\left.\quad-2 k^{2} \Gamma_{-} K_{-}\left(\left(\Gamma_{-}-1\right) n_{-}^{\Gamma_{-}-2} n_{-}^{\prime 2}+n_{-}^{\Gamma_{-}-1} n_{-}^{\prime \prime}\right)\right] \\
=\frac{-e^{2}}{\varepsilon}\left(n_{+}-n_{-}\right)^{3} .
\end{aligned}
$$

As is the case for approach I, the two equations are then solved simultaneously to obtain the coefficients $c_{ \pm s \rho}$ with $s=1,2 \ldots N$. For the first order, one respectively recovers (87) and (88) without the second $c_{+1 \rho}$ (i.e., $c_{+1 \rho}=m_{-} / m_{+}$). Hence, one obtains the dispersion relation (89) without the usually rejected Eq. (90). In Appendix II we briefly give the expressions of the coefficients up to order two only since they are very long. However, we give them up to order five for the electron-positron plasma.

\section{Brief discussion on the dispersion relation}

\section{Cold plasma}

If $T_{+} \approx 0 \approx T_{-}$, then $v_{s_{-}} \approx 0 \approx v_{s+}$ and (89) becomes a quadratic equation $\omega^{4}-\omega^{2}\left(\omega_{+}^{2}+\omega_{-}^{2}\right)=0$ with roots $\omega^{2}=0$ (trivial solution) and

$$
\omega^{2}=\omega_{+}^{2}+\omega_{-}^{2} \equiv \omega_{i e}^{2} \text {. }
$$

Equation (93) tells us that both electrons and ions are oscillating around their equilibrium positions with frequency $\omega_{i e}$ without propagating. The results obtained here are the same as those for $k \approx 0$.

\section{Ions stand still}

Substituting $\omega_{+}=0=v_{s+}$ into (89) one recovers the dispersion relation (69) obtained when ions are not moving (together with the trivial solution $\omega=0$ ).

3. Dust plasma and bucky balls

Similarly putting $\omega_{-}=0=v_{s-}$ into (89) yields the trivial solution $\left(\omega^{2}=0\right)$ and the dispersion relation for the case when the negative particles stand still:

$$
\omega^{2}=\omega_{+}^{2}+k^{2} v_{s+}^{2}
$$

This is the case when the mass of the negative particles is much higher than the ion mass. This was long considered as an unphysical situation. However, in the tails of comets and in the interplanetary space e.g., the dust particles are sometimes negatively charged due to electrons which are attached to them. Those dust particles may easily have masses of $10^{8}$ a.m.u and accumulate hundreds or thousands of electrons [12].

In the laboratory this situation may, e.g., occur using bucky balls or fullerenes, i.e., carbon sixty (or seventy, ...) which are constituted by 60 carbon atoms. Hence their mass is $720 \mathrm{amu}$ (respectively $840 \mathrm{amu}$ for 70 carbon atoms). The fullerenes have the property that they may attach an electron so that they become negatively charged. The frequencies are of course much lower than for an "ordinary plasma" [13].

\section{Electron-positron plasma}

Again the interest in this exotic plasma dates from the last decennium. Photons with energy higher than $1 \mathrm{MeV}$ may disintegrate in an electron and a positron (rest energy $511 \mathrm{keV})$. Around pulsars one may have a plasma of extremely high temperature (over $10^{10} \mathrm{~K}$ ), which allows the photons to disintegrate. As the density of photons is extremely high the density of electrons and positrons is extremely high as well (the density is in the range of $10^{32} \mathrm{~m}^{-3}$ ) [14,15]. For an electron-positron plasma we have $m_{-}=m_{+}$and thus $\omega_{-}=\omega_{+}$. Substituting this in (89) yields $\omega^{2}=2 \omega_{-}^{2}$ together with $\omega^{2}=k^{2}\left(v_{s-}^{2}+v_{s+}^{2}\right)$. This result is surprising: the electric waves and the pressure waves are decoupled and exist each on their own. We may interpret this physically as follows:

(a) For the equation $\omega^{2}=2 \omega_{-}^{2}$.

The electron and the positron oscillate symmetrically around the equilibrium situation. As a positron moving to the right is electrically the same as an electron moving to the left, the situation is electrically the same as if twice as many electrons are oscillating which explains the factor 2 . The associated pressure waves however cancel each other leaving the total pressure unaltered, so that this dispersion relation does not depend on $v_{s-}^{2}$ or $v_{s+}^{2}$. However, the higher order coefficients do depend on the sound velocity (see Appendix II), which is surprising. In fact this feature happens because the even coefficients are the same for electrons and positrons, while the odd coefficients are opposite. Hence from second order on variations in the total density happen. 
(b) For the equation $\omega^{2}=k^{2}\left(v_{s-}^{2}+v_{s+}^{2}\right)$.

The electrons and the positrons move together, hence no electric perturbation occurs and only terms corresponding to the pressure wave occur. Note that the temperature of the positrons does not have to be equal to the one of the electrons.

An estimate of the ratio of both frequencies, provided the temperatures are assumed to be the same, is given by

$\frac{\omega_{-}}{k v_{s-}}=\frac{e}{k} \sqrt{\frac{n_{0}}{\varepsilon \Gamma k_{B} T}}$.

With $n_{0}=10^{32} \mathrm{~m}^{-3}$ and $T=10^{10} \mathrm{~K}$ we find $\omega_{-} /\left(k v_{s-}\right)=$ $10^{9} / k$. Hence both frequencies are comparable for wavelengths of about $10^{-8} \mathrm{~m}$, for larger wavelengths the plasma frequency is the larger one.

(c) Graphical representation.

Sketching the graphs of $\omega^{2}=2 \omega_{-}^{2}$ and $\omega^{2}=2 k^{2} v_{s-}^{2}$ on the same graph (see Figure 2), one obviously sees that they meet at the point $\left(\omega_{m}, k_{m}\right)$, where $k_{m}$ is given by (71) and $\omega_{m}$ is the the value of the frequency when $k=k_{m}=\left(\Lambda_{D-} \sqrt{\Gamma_{-}}\right)^{-1}$. It is also interesting to see that, at the same point, the two graphs cross the graph of $\omega^{2}=\omega_{-}^{2}+k^{2} v_{s-}^{2}$, which was considered previously in section 3.3. This gives the parameter $k_{m}$ a much stronger basis as it can be used for comparison purposes.

\section{Conclusion}

In this study we have considered perturbations of ideal unmagnetized plasmas which were represented by systems of nonlinear partial differential equations. We reduced each system to a single nonlinear equation in one unknown, the combined variable $\chi=\omega t-\boldsymbol{k} \cdot \boldsymbol{r}$. The reduction process was done using either the first or the second or the third procedure. These procedures reduce the system respectively to a first and a second order differential equation and a fully integrated equation. Of the three procedures, the second one is simpler and faster than the others. Thus it turns out that the higher the order of the reduced differential equation, the simpler the way to solve the Fourier analysis, contrary to our original expectations. The higher orders were obtained using mathematica. The solution is expressed in terms of the following parameters: the equilibrium quantities $\left(\omega_{ \pm}, v_{s \pm}, \Gamma_{ \pm}, n_{0}\right)$, the amplitude of the linearized solution $\left(A_{-}\right)$, and the wave parameters $(\omega, k)$.

Callebaut's method of Fourier analysis was used, i.e., only one family of $(\omega, k)$ is considered (i.e., one linear term and all its related higher order terms). If two or more $(\omega, k)$ are considered (with different phase velocity) then interference terms between the families are needed in addition.

In cold plasma we noted that, the series in exponentials converges with amplitudes less than $\mathrm{e}^{-1}$ of the initial density. However with sinusoidal waves, corresponding to experimental situations, this amplitude is doubled, i.e., up to $78 \%$ of the initial density. In sound waves, the series did not converge at all. The nonlinear approach does not work for sound waves. This is due to the fact that the dispersion relation yields a straight line through the origin $\left(\omega=v_{s} k\right)$ so that $\left(\partial_{t t}^{2}-v_{s}^{2} \Delta\right)$ is always zero for all $(\omega, k)$ and all $\rho$ : to compen- sate this the coefficient is required to be infinite and thus there can be no convergence. For the case of plasma with electronic pressure, the series converged with amplitudes smaller than $1 /$ e for exponentials (respectively smaller than $2 / \mathrm{e}$ for sinusoidal waves). As the natural oscillations have a very limited amplitude (the total thermal energy is an upper bound; making use of table II we have estimated that the amplitude $A_{-}$for oscillations based on spontaneous fluctuations should be at most $10^{-5}$ ) one should use very strong external fields to verify these results experimentally in a plasma.

We have elaborated some nonlinear terms for the case when both the electron and ion pressure are taken into account. This is relevant in particular for certain exotic plasmas which are studied since last decennium: comet tails, fullerenes and electron-positron plasmas. In fact the electron-positron plasma will be considered fully in a forthcoming paper including the magnetic contribution. In addition we have derived and commented on the dispersion relations of various waves and approximations.

\section{Acknowledgement}

It is a pleasure to thank Prof. Dr. W. Malfliet (UA, Antwerp, Belgium) for comments. One of us (G. K. K) is greatly indebted to the Flemish Interuniversity Council (VLIR, Brussels, Belgium) for a Ph.D. grant in the SUAVLIR program.

\section{APPENDICES}

\section{Coefficients of the higher order terms for the case of} plasma with pressure of electrons

In this appendix we give some of the results obtained for particle density, velocity and potential with $\omega_{\Omega}=$ $\left(1+\Gamma_{-}\right) k^{2} v_{s-}^{2} / \omega_{-}^{2}=k^{2} \Gamma_{-}\left(1+\Gamma_{-}\right) \Lambda_{D-}^{2}, \quad$ where $\Lambda_{D-}^{2}=$ $\varepsilon K_{-} n_{0}^{\Gamma_{-}} / e^{2} n_{0}^{2}$. We calculated the coefficients up to order 15 , but give only the first five here.

\section{I.1. Coefficients in the particle density of $n / n_{0}$ (normalized particle-density)}

$b_{-1 \rho}=1$,

$b_{-2 \rho}=2+\frac{2}{3} \omega_{\Omega}$,

$b_{-3 \rho}=\frac{9}{2}+\frac{3}{4} \omega_{\Omega}^{2}+\left(\frac{27}{8}+\frac{3 \Gamma_{-}}{16}\right) \omega_{\Omega}$,

$b_{-4 \rho}=\frac{32}{3}+\frac{28}{27} \omega_{\Omega}^{3}+\omega_{\Omega}^{2}\left(6+\frac{5 \Gamma_{-}}{9}\right)$

$$
+\omega_{\Omega}\left(\frac{194}{15}+\frac{11 \Gamma_{-}}{9}+\frac{2 \Gamma_{-}^{2}}{45}\right)
$$

$$
\begin{aligned}
b_{-5 \rho}= & \frac{625}{24}+\frac{2075}{1296} \omega_{\Omega}^{4}+\omega_{\Omega}^{3}\left(\frac{2425}{216}+\frac{575 \Gamma_{-}}{432}\right) \\
& +\omega_{\Omega}^{2}\left(\frac{18385}{576}+\frac{8975 \Gamma_{-}}{1728}+\frac{1795 \Gamma_{-}^{2}}{6912}\right) \\
& +\omega_{\Omega}\left(\frac{6395}{144}+\frac{265 \Gamma_{-}}{48}+\frac{205 \Gamma_{-}^{2}}{576}+\frac{5 \Gamma_{-}^{3}}{576}\right) .
\end{aligned}
$$


I.2. Coefficients in the velocity (in units $-\omega / k$ )

$b_{-1 v}=1$,

$$
\begin{aligned}
b_{-2 v}= & +\frac{2 \omega_{\Omega}}{3}, \\
b_{-3 v}= & \frac{3}{2}+\left(\frac{49}{24}+\frac{3 \Gamma_{-}}{16}\right) \omega_{\Omega}+\frac{3 \omega_{\Omega}^{2}}{4}, \\
b_{-4 v}= & \frac{8}{3}+\left(\frac{331}{60}+\frac{61 \Gamma_{-}}{72}+\frac{2 \Gamma_{-}^{2}}{45}\right) \omega_{\Omega} \\
& +\left(\frac{73}{18}+\frac{5 \Gamma_{-}}{9}\right) \omega_{\Omega}^{2}+\frac{28 \omega_{\Omega}^{3}}{27}, \\
b_{-5 v}= & \frac{125}{24}+\left(\frac{10441}{720}+\frac{26 \Gamma_{-}}{9}+\frac{769 \Gamma_{-}^{2}}{2880}+\frac{5 \Gamma_{-}^{3}}{576}\right) \omega_{\Omega} \\
& +\left(\frac{9217}{576}+\frac{6623 \Gamma_{-}}{1728}+\frac{1795 \Gamma_{-}^{2}}{6912}\right) \omega_{\Omega}^{2} \\
& +\left(\frac{587}{72}+\frac{575 \Gamma_{-}}{432}\right) \omega_{\Omega}^{3}+\frac{2075 \omega_{\Omega}^{4}}{1296} .
\end{aligned}
$$

I.3. Coefficients in the potential (in units $-e n_{0} / k^{2} \varepsilon$ ).

$$
\begin{aligned}
b_{-1 \varphi}= & 1, \\
b_{-2 \varphi}= & \frac{1}{2}+\frac{\omega_{\Omega}}{6}, \\
b_{-3 \varphi}= & \frac{1}{2}+\left(\frac{3}{8}+\frac{\Gamma_{-}}{48}\right) \omega_{\Omega}+\frac{\omega_{\Omega}^{2}}{12}, \\
b_{-4 \varphi}= & \frac{2}{3}+\left(\frac{97}{120}+\frac{11 \Gamma_{-}}{144}+\frac{\Gamma_{-}^{2}}{360}\right) \omega_{\Omega} \\
& +\left(\frac{3}{8}+\frac{5 \Gamma_{-}}{144}\right) \omega_{\Omega}^{2}+\frac{7 \omega_{\Omega}^{3}}{108}, \\
b_{-5 \varphi}= & \frac{25}{24}+\left(\frac{1279}{720}+\frac{53 \Gamma_{-}}{240}+\frac{41 \Gamma_{-}^{2}}{2880}+\frac{\Gamma_{-}^{3}}{2880}\right) \omega_{\Omega} \\
& +\left(\frac{3677}{2880}+\frac{359 \Gamma_{-}}{1728}+\frac{359 \Gamma_{-}^{2}}{34560}\right) \omega_{\Omega}^{2} \\
& +\left(\frac{97}{216}+\frac{23 \Gamma_{-}}{432}\right) \omega_{\Omega}^{3}+\frac{83 \omega_{\Omega}^{4}}{1296} .
\end{aligned}
$$

\section{The coefficients when both ions and electrons are moving}

In this appendix we give the coefficients (up to second order terms) in the expressions of the particle densities when both ions and electrons in the plasma are moving. We put $c_{-1 \rho}=1$. (In the case that the negative particles have a much larger mass than the ions (comet-tail particles, fullerenes) and that one considers the limit that they are standing still, then one has to put $c_{-1 \rho}=0$ and $c_{+1 \rho}=1$ ) with the following solution of the dispersion relation (89)
(+ sign in front of the square root)

$$
\begin{aligned}
\omega^{2}= & \frac{1}{2}\left\{\omega_{-}^{2}+\omega_{+}^{2}+k^{2}\left(v_{s-}^{2}+v_{s+}^{2}\right) .\right. \\
& +\left\{\left[\omega_{-}^{2}+\omega_{+}^{2}+k^{2}\left(v_{s-}^{2}+v_{s+}^{2}\right)\right]^{2} .\right. \\
& \left.\left.-8 k^{2}\left(\omega_{+}^{2} v_{s-}^{2}+\omega_{-}^{2} v_{s+}^{2}\right)\right\}^{\frac{1}{2}}\right\}
\end{aligned}
$$

and obtained:

$$
\begin{aligned}
& c_{+1 \rho}=\frac{\omega_{+}^{2}\left(\omega_{-}^{2}-k^{2} v_{s-}^{2}\right)}{\omega_{-}^{2}\left(-\omega^{2}+\omega_{+}^{2}+k^{2} v_{s+}^{2}\right)}, \\
& c_{-2 \rho}=\frac{\Psi_{1}+2 \omega_{+}^{2} \Psi_{2}}{\Psi_{3}}, \\
& c_{+2 \rho}=\frac{\Phi_{1}+2 \omega_{-}^{2} \Phi_{2}}{\Phi_{3}}
\end{aligned}
$$

where

$$
\begin{aligned}
\Psi_{1}= & \omega_{+}^{4}\left\{\omega_{-}^{2}\left(c_{+1 \rho}-1\right)^{2}+2\left[\omega^{2}+k^{2}\left(\Gamma_{-}-1\right) v_{s-}^{2}\right]\right\} \\
& -4 k^{2} \omega^{2} \omega_{-}^{2}\left(1+\Gamma_{+}\right) c_{+1 \rho}^{2} v_{s+}^{2}
\end{aligned}
$$

$$
\begin{aligned}
\Psi_{2}= & 2 \omega^{2}\left[-2 \omega^{2}+k^{2}\left(1-\Gamma_{-}\right) v_{s-}^{2}+2 k^{2} v_{s+}^{2}\right] \\
& +\omega_{-}^{2}\left\{2 \omega^{2}\left(-1+c_{+1 \rho}\right)+2 k^{2} v_{s+}^{2}\right. \\
& \left.+c_{+1 \rho}^{2}\left[\omega^{2}+k^{2}\left(-3+\Gamma_{+}\right) v_{s+}^{2}\right]\right\}
\end{aligned}
$$$$
\Psi_{3}=2 \omega_{+}^{2}\left\{\omega^{2}\left(\omega_{-}^{2}+\omega_{+}^{2}\right)-2 k^{2}\left(\omega_{+}^{2} v_{s-}^{2}+\omega_{-}^{2} v_{s+}^{2}\right)\right.
$$$$
\left.+4 \omega^{2}\left[-\omega^{2}+k^{2}\left(v_{s-}^{2}+v_{s+}^{2}\right)\right]\right\},
$$

$$
\begin{aligned}
\Phi_{1}= & -4 k^{2} \omega^{2}\left(1+\Gamma_{-}\right) \omega_{+}^{2} v_{s-}^{2}+\omega_{-}^{4}\left\{\omega_{+}^{2}\left(c_{+1 \rho}-1\right)^{2}\right. \\
& \left.+2 c_{+1 \rho}^{2}\left[\omega^{2}+k^{2}\left(\Gamma_{+}-1\right) v_{s+}^{2}\right]\right\},
\end{aligned}
$$$$
\Phi_{2}=\omega_{+}^{2}\left[\omega^{2}+2 \omega^{2} c_{+1 \rho}+k^{2}\left(\Gamma_{-}-3\right) v_{s-}^{2}\right.
$$$$
\left.-2 c_{+1 \rho}^{2}\left(\omega^{2}-k^{2} v_{s-}^{2}\right)\right]
$$$$
+2 \omega^{2} c_{+1 \rho}^{2}\left[-2 \omega^{2}+k^{2}\left(2 v_{s-}^{2}+\left(1-\Gamma_{+}\right) v_{s+}^{2}\right)\right],
$$

$$
\begin{aligned}
\Phi_{3}= & 2 \omega_{-}^{2}\left(\omega_{+}^{2}\left(\omega^{2}-2 k^{2} v_{-s}^{2}\right)\right. \\
& +\omega_{-}^{2}\left(\omega^{2}-2 k^{2} v_{s}^{2}\right) \\
& \left.+4 \omega^{2}\left(-\omega^{2}+k^{2} v_{-s}^{2}+k^{2} v_{s}^{2}\right)\right) .
\end{aligned}
$$

Note that putting the mass of the negative particles infinite would yield $c_{+1 \rho}$ infinite. Clearly for that case one has to put $c_{-1 \rho}=0$ and $c_{+1 \rho}=1$ and make the required interchanges in the formulae.

When one investigates the case of electron-positron plasma $\left(\omega_{+}=\omega_{-}\right)$, with the temperature of electrons assumed to be equal to that of the positrons (implying 
that $\Gamma_{+}=\Gamma_{-}$and $\left.v_{s+}^{2}=v_{s-}^{2}\right)$ then one obtains the following results, taking $\omega^{2}=2 \omega_{-}^{2}$ and $c_{-1 \rho}=1$ :

$$
\begin{aligned}
c_{+1 \rho}= & -1 \\
c_{ \pm 2 \rho}= & \frac{3 \omega_{-}^{2}+k^{2}\left(\Gamma_{-}-1\right) v_{s-}^{2}}{2\left(\omega_{-}^{2}-k^{2} v_{s-}^{2}\right)}, \\
c_{ \pm 3 \rho}= & \mp \frac{45 \omega_{-}^{2}+3 k^{2}\left(-1+7 \Gamma_{-}\right) v_{s-}^{2}}{16\left(\omega_{-}^{2}-k^{2} v_{s-}^{2}\right)} \\
c_{ \pm 4 \rho}= & \frac{1}{48\left(\omega_{-}^{2}-k^{2} v_{s-}^{2}\right)^{3}}\left\{255 \omega_{-}^{6}\right. \\
& +k^{2}\left[-345+2 \Gamma_{-}\left(125+\Gamma_{-}\left(12+\Gamma_{-}\right)\right)\right] \omega_{-}^{4} v_{s-}^{2} \\
& +k^{4}\left[117+\Gamma_{-}\left(-254+\Gamma_{-}\left(51+8 \Gamma_{-}\right)\right)\right] \omega_{-}^{2} v_{s-}^{4} \\
& \left.-k^{6}\left(-1+\Gamma_{-}\right)\left(-3+\Gamma_{-}\left(49+4 \Gamma_{-}\right)\right) v_{s-}^{6}\right\} \\
\left.c_{ \pm 5 \rho}\right\} & \mp \frac{1}{2304\left(\omega_{-}^{2}-k^{2} v_{s-}^{2}\right)^{3}}\left\{24525 \omega_{-}^{6}\right. \\
& -5 k^{2}\left[4959-2 \Gamma_{-}\left(2879+22 \Gamma_{-}\left(12+\Gamma_{-}\right)\right)\right] \omega_{-}^{4} v_{s-}^{2} \\
& +5 k^{4}\left[591-\Gamma_{-}\left(4940-\Gamma_{-}\left(1413+176 \Gamma_{-}\right)\right)\right] \omega_{-}^{2} v_{s-}^{4} \\
& \left.-5 k^{6}\left[9-\Gamma_{-}\left(238-\Gamma_{-}\left(1281+88 \Gamma_{-}\right)\right)\right] v_{s-}^{6}\right\} .
\end{aligned}
$$

However, the detailed electron-positron plasma will be considered in a forthcoming paper including the magnetic terms in the basic equations. On the other hand, if the $\omega^{2}$ used was the one involving the minus sign in front of the square root given in relation (95), one would have obtained $c_{-s \rho}$ 's which gives infinity when the electron-positron plasma case is considered. This implies that our expression for the particle density becomes divergent. In fact this corresponds to the case of sound waves (see section 3.2) and our nonlinear Fourier analysis using $\chi$ is not suitable then. However, when the electron and ion masses are different this divergency does not occur.

\section{References}

1. Callebaut, D. K., "Lineaire en niet-lineaire perturbaties in hydro-, magneto- en gravitodynamica," (Simon Stevin 45, Ghent, 1972), pp. $1-315$.

2. Malfliet, W., Am. J. Phys. 60, 650 (1992); Malfliet, W. and Hereman, W., Physica Scripta 54, 569 (1996); Malfliet, W. and Wieërs, E., J. Plasma Phys. 56, 441 (1996).

3. Hereman, W. et al., J. Phys. A-Math. Gen. 19, 607 (1986); Hereman, W., and Nuseir, A., Math. Comput. Simulat. 43, 13 (1997); Baldwin, D. et al., J. Symb. Comput., submitted.

4. Verheest, F., J. Plasma Phys. 39, 71 (1988); Verheest, F., Plan. Space Sci. 40, 1 (1992); Verheest, F. and Buti, B., J. Plasma Phys. 47, 15 (1992); Verheest, F., ibid. 47, 25 (1992); Verheest, F., Space Sci. Rev. 77, 267 (1996).

5. Khater, Ibrahim, A. K., Ibrahim, R. S., Shamardan, A. B. and Callebaut, D. K., IMA J. Appl. Math. 58, 51 (1997); Khater, A. K., El-Kalaawy, O. H. and Callebaut, D. K., Physica Scripta 58, 545 (1998); Khater, A. K., Callebaut, D. K. and Kamel, E. S., Solar Phys. 178, 285 (1998); Khater, A. K., Callebaut, D. K., Abdalla, A. A. and Sayed, S. M., Chaos, Solitons Fractals 10, 1309 (1999); Khater, A. K., Callebaut, D. K. and Seadawy, A. R., Physica Scripta 62, 353 (2000); Khater, A. K., Callebaut, D. K., Malfliet, W. and Seadawy, A. R., ibid. 64, 533 (2001).

6. Amiranashvili, Sh., Yu, M. Y., Stenflo, L., Brodin, G., and Servin, M., Phys. Rev. E 66, 046403 (2002).

7. Callebaut, D. K. and Tsintsadze, N. L., Physica Scripta 50, 283 (1994); Callebaut, D. K. and Tsintsadze, N. L., Physica Scripta 52, 565 (1995)

8. Callebaut, D. K., in "Workshop II on (Nonlinear) Stability," (Edited by Callebaut, D. K., Mertens, R. A. and Malfliet, W.) (UIA Press, Antwerp, 1980), p. 166; Callebaut, D. K., in Proc. 10th European School on Plasma Physics. (Edited by N. L. Tsintsadze) (World Scientific, Singapore, 1991), p. 286.

9. Callebaut, D. K. "Introduction to Stability Problems in Fluid Mechanics and Plasma Physics," (UIA Press, Antwerp, 1986, revised 2002), pp. 1-190.

10. Wolfram, S., "The Mathematica Book," (4th edition. Mathematica version 4.) (Wolfram Media, Urbana-Champaign, Illinois and Cambridge University Press, London, 1999).

11. Mathews, J. and Walker, R. L., "Mathematical Methods of Physics," (2nd edition) (W. A. Benjamin, INC, New York, 1972), p. 45.

12. de Angelis, U., Physica Scripta 45, 465 (1992); Northrop, T. G., Physica Scripta 45, 475 (1992); Shukla, P. K., Physica Scripta 45, 504 (1992); Verheest, F. Phys. Plasmas 5, 1225 (1998).

13. Strele, D., Winkler, C., Krumm, P. and Schrittwieser, R., Plasma Sources Sci. Technol. 5, 603 (1996).

14. Callebaut, D. K., Tsintsadze, L. N., and Tsintsadze, N. L., in Proc. 32nd Liège International Astrophysical Colloquium. (Edited by A. Noels, D. Fraipont-Caro, M. Gabriel, N. Grevesse and P. Demarque) (Université de Liège, Liège, 1995), p. 233.

15. Tsintsadze, L. N., Callebaut, D. K. and Tsintsadze, N. L., J. Plasma Phys. 55, 407 (1996). 